\title{
Étude des volutes spirales des pompes centrifuges, en tenant compte de la répartition des pressions statiques
}

\section{The design of the volute of centrifugal pumps, taking into consideration the static pressure distributions}

\author{
Par le Professeur-adjoint Aziz ERGIN \\ SERVIGE DES MACHINES HYDRAULQUES \\ VNIVERSTÉ TECHNLUE D'ISTANBUE
}

\begin{abstract}
Le présent mémoire est une étude thérique sur le probleme suivant: comment obtenir une répartition uniforme des pressions statiques antour de la périphérie de la rone dans la volute spirale d'une pompe centrifuge? Pour cette étude, on a assimilé le débit sortant de la route $\dot{a}$ un jet périphérique qui se mélange $\dot{a}$ l'écoulement déjà existant dans la volute spirale.

Par ailleurs, on considere l'aire de section de la volute spirale comme étant la somme de dear aires distinctes, dont l'une est occupée par l'écoulement de la volute et l'autre contient le jet périphérique sortant de la rone.

Les résultats de deux tripériences exécutées ave la même pompe centrifuge et dans les mèmes conditions de fonctionnement, mais, dans le second cas, avec the volute spirale spécialement étudiese afin d'obtenir une répatition uniforme des pressions statiques, nous amenent i conclure que la méthode de calcul caposée dans ce mémoire fournit une amélioration do $2 \%$ du rendement, ainsi qu'une répartition plus uniforme des pressions statiques dans: la volute spirale.
\end{abstract}

\begin{abstract}
This paper is a theoretical study of how to obtain a uniform static pressure in the volute of a centrifugal pump, around the periphery of the impeller. For this, the outfow of the impeller is considered as a circumferential jet mixing with the flow alread! existing in the volute.

The cross-sectional area of the volute is considered as the sum of two separate areas, the area where the volute flow exists and the area occupied by the circumferential jot flowing out of the impeller.

The results of two experiments with the sume centrifugal pump, under the same working conditions, but the second with a volute calculated to obtain a constant static pressure distribution show that this method of the calculation gave an advantage of $2 \%$ in the efficiency and a more uniform static pressure distribution around the volute.
\end{abstract}

\section{TABLE DES NOTATIONS}

Q Débit en une section quelconque de la volute spirale (ne comprend pas le débit dans la section du jet).

$Q_{p}$ Débil nominal de la pompe.

$Q_{1}$ Débit entrant dans la volute au point où $0=0$.

$\eta$ Rendement.

H Hauteur totale.

$Q$ Aire d'une section quelconque de la volute (ne comprend pas l'aire de section du jet).

$\Omega_{j}$ Aire de section du jet.

a Angle autour de la volute, mesuré dans le sens de l'écoulement.

$\mathrm{V}$ Vitesse moyenne sur l'aire $\Omega$. 
c Composante tangentielle de la vitesse absolue du jet d'eau au rayon moyen $\left(\mathbf{R}_{a v}\right)$.

$V_{t}$ Composante tangentielle de la vitesse absolue à la sortie de la roue.

C. Constante d'intégration.

$\mathrm{R}_{a v}$ Rayon moyen pris par rapport à l'aire $\Omega$.

$\alpha_{1}$ Angle obtenu en reliant les points $E_{1}$ et $F_{1}$ au centre de la roue.

$\mathrm{E}_{1}$ Point à la sortie de la roue où $0=0$ pour la partie de la volute considérée.

$F_{1}$ Point atteint sur la partie extérieure de la volute par une particule d'eau partant du point $\theta=0$ de la roue.

$k$ Coefficient caractérisant la variation de la valeur de l'angle $\alpha$ autour de la périphérie de la volute.

$a$ Coefficient caractérisant le taux de mélange du jet avec le débit dans la volute.

\section{INTRODUCTION}

Il est très difficile d'obtenir une pression statique constante autour de la périphérie d'une pompe centrifuge, surtout lorsque celle-ci fonctionne avec un débit autre que le débit nominal. On a pu constater qu'en général, à des débits inférieurs au débit nominal, la pression statique dans la volute spirale augmente à mesure que l'on approche de la sortie de la spirale, et que dans le cas contraire, c'est-à-dire pour des débits supérieurs à la valeur nominale, elle diminue.

Par ailleurs, on a aussi constaté que, même pour le débit nominal où la pression statique était supposée constante, des fluctuations considérables se superposaient à la répartition générale des pressions (voir les expériences $n^{\circ} 1$ et $\mathrm{n}^{\circ} 3$ de la présente série).

Le présent mémoire contient une analyse des phénomènes se produisant dans la volute, proposant également un procédé théorique d'étude pouvant être employée pour celle-ci, compte tenu de l'influence de la variation de l'aire de section, celle de sa forme, et la nature de l'écoulement provenant de la roue. Finalement, le mémoire fait état des résultats de trois séries d'expériences effectuées sur le même problème au Laboratoire d'Hydraulique de l'Université de Cambridge.

L'une des conclusions les plus intéressantes est probablement qu'il est impossible d'obtenir une répartition constante des pressions sur la périphérie de la volute si les aires de section à la sortie de celle-ci sont grandes .

Bien que la formule donnée à la suite de la présente étude pour calculer la forme de la volute soit en elle-même compliquée, son emploi s'impose néanmoins pour des sections successi- ves de la volute, étant donné que les paramètres $k$ et $a$ de l'équation (24), qui sont supposés constants, ne sont en fait pas indépendants de 0 . Comme nous le verrons, la formule exige certaines données qui ne peuvent être obtenues que par voie expérimentale : données sur la répartition de l'eau sortant de la roue et sur l'influence de la forme de la volute aussi bien en ce qui concerne sa section normale à l'écoulement que la courbe extérieure située dans le plan de l'écoulement. Les calculs deviennent de ce fait encore plus laborieux.

D'autre part, la formule tient compte de tous les points suivants :

1. Le fait que l'angle d'écoulement de l'eau à la sortie de la roue est fonction de la vitesse. radiale;

2. L'influence du jeu à la languette;

3. L'allure de la courbure de la paroi extérieure de la volute.

4. L'influence de la forme de la section de la volute;

5. L'influence de contractions à l'extrémité de la volute proprement dite.

Le seul facteur dont la formule ne tient pas compte est la composante de vitesse radiale de l'eau sortant de la roue au point où elle atteint la paroi extérieure de la volute. Cette composante de vitesse étant beaucoup plus petite que la composante de vitesse radiale à la sortie même de la roue, est considérée comme étant perdue, bien qu'en fait une petite partie de celle-ci soit rete- 
nue en réalité, s'ajoutant à la composante tangentielle à travers la courbure des parois de la volute. Si l'on désire toutefois en tenir compte dans les calculs, on peut facilement l'ajouter à $\ll c »$, qui est la composante de vitesse tangentielle au rayon moyen.

Dans une pompe centrifuge, l'eau sortant de la roue se présente sous la forme d'une série de jets. Comme le montre la figure 1, nous avons con-

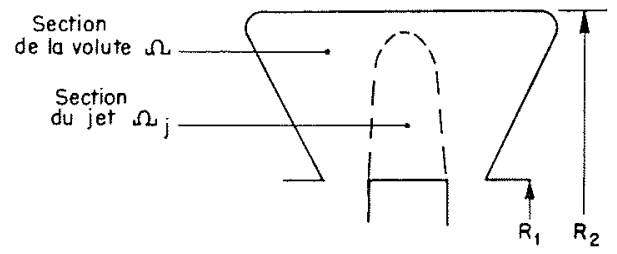

FII. 1

sidéré deux aires indépendantes l'une de l'autre dans une section quelconque de la volute, soit :
1. La section de la volute $\alpha$, contenant le débit dans celle-ci;

2. La section du jet $\Omega_{j}$, contenant le jet. Le sens de l'écoulement dans celle-ci est sensiblement le même que celui de l'eau à la sortie de la roue.

Les trois expériences sont eflectuées sur une pompe centrifuge spécialement conçue afin de permettre facilement toute modification de la section de la volute. Nous avons accepté une volute standard pour la première expérience, mais nous en avons calculé une spécialement pour la seconde et la troisième, en nous basant sur la formule obtenue grâce à la partie théorique de la présente étude.

On constate, d'après les résultats de la troisième expérience, que le rendement s'est amélioré de $2 \%$ par rapport au profil de volute classique, et que la répartition des pressions statiques autour de la volute est devenue plus uniforme.

\section{INFLUENCE D'UNE RÉPARTITION NON UNIFORME DES PRESSIONS STATIQUES AUTOUR DE LA ROUE}

La pression statique dans la volute est un des lacteurs principaux déterminant le débit et la répartition de l'écoulement à la sortie de la roue. Dans le cas où la répartition des pressions autour de la périphérie de la volute est uniforme, les débits d'eau sortant de tous les points de la roue seront égaux. Si nous exprimons le débit correspondant à l'angle $d \oplus$ par $d q$, nous avons :

$$
d q=\frac{Q_{p}}{2 \pi} \cdot d \theta
$$

Désignons par $A B$ (fig. 2) l'arc de la périphérie

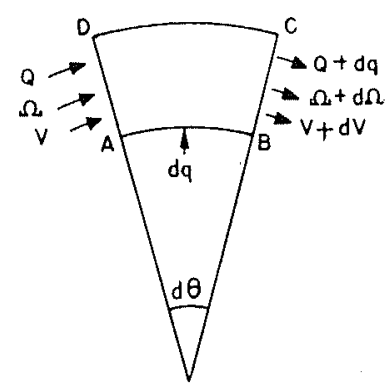

Fig. 2

de la roue correspondant à l'angle $d 0$, et par «volume $A B C D$ » le volume correspondant de la volute contenant également le volume occupé par le jet (voir fig. 1).

De fait, le débit $d q$ sortant de la roue par voie de l'are $A B$ continuera d'abord à s'écouler dans la section du jet, se mélangeant plus tard avec l'écoulement dans la volute.

Supposons, pour simplifier, que l'eau pénétrant dans l'écoulement de la volute dans le voIume $A B C D$ est égal au débit sortant de la roue $d q$. Si nous désignons la section, le débit, et la vitesse moyenne à la section $A B$ par $\Omega, Q$ et $v$, ces grandeurs deviendront respectivement $\Omega+d \Omega$, $\mathrm{Q}+d q$ et $v+d v$ à la section $\mathrm{BC}$.

En admettant que « la pression statique dans la volute varie radialement selon une même loi donnée, quelle que soit la valeur de $\theta »$, et en négligeant la courbure nous pouvons faire intervenir la loi des quantités de mouvement, ce qui nous donne :

$$
\mathrm{Q} \cdot v+d q \cdot c=(\mathrm{Q}+d q)(v+d v)
$$

$c$ étant la composante langentielle de la vitesse absolue du jet d'eau avant qu'il ne se mélange avec l'écoulement dans la volute.

Nous tirons de Ja définition de la vitesse moyenne :

$$
\frac{\mathrm{Q}}{\Omega}=v
$$


et :

$$
\frac{\mathrm{Q}+d q}{\Omega+d \Omega}=v+d v
$$

d'où, en intégrant l'équation (1) :

$Q_{1}$ étant le débit à la languette.

$$
Q=\frac{Q_{p}}{2 \pi}-\theta+Q_{1}
$$

Nous obtenons, des équations (1), (2), (3) et (4):

$$
\frac{\mathrm{Q}^{2}}{\Omega}+c \frac{\mathrm{Q}_{p}}{2 \pi} d \theta=\left(\mathrm{Q}+\frac{\mathrm{Q}_{p}}{2 \pi} d \theta\right) \frac{\mathrm{Q}+\left(\mathrm{Q}_{p} / 2 \pi\right) d \theta}{\Omega+d \Omega}
$$

Substituant la valeur de $Q$ de l'équation (5) :

$$
\frac{\left[\left(\mathrm{Q}_{p} / 2 \pi\right) \theta+\mathrm{Q}_{1}\right]^{2}}{\Omega}+c \frac{\mathrm{Q}_{p}}{2 \pi} d \theta=\frac{\left[\left(\mathrm{Q}_{n} / 2 \pi\right) \theta+\mathrm{Q}_{1}+\left(\mathrm{Q}_{p} / 2 \pi\right) d \theta\right]^{2}}{\Omega+d \Omega}
$$

En négligeant le deuxième ordre des petites quantités, nous pouvons écrire :

c'est-à-dire :

$$
\left(\frac{Q_{p}}{2 \pi} \theta+Q_{1}\right)^{*} \frac{d \Omega}{\Omega}+c \frac{Q_{n}}{2 \pi} \Omega d \theta=2 \quad\left(\frac{Q_{p}}{2 \pi} \theta+Q_{1}\right) \frac{Q_{n}}{2 \pi} d \theta
$$

$$
\left(0+\frac{2 \pi \mathrm{Q}_{1}}{\mathrm{Q}_{p}}\right)^{2} \frac{d \Omega}{\Omega}+\frac{2 \pi c}{\mathrm{Q}_{p}} \Omega d \theta=2\left(\theta+\frac{2 \pi \mathrm{Q}_{1}}{\mathrm{Q}_{p}}\right) d \theta
$$

Posons :

$$
\left\{\begin{array} { l } 
{ \theta + \frac { 2 \pi Q _ { 1 } } { Q _ { p } } = x , } \\
{ d \theta = d x }
\end{array} \left\{\begin{array}{l}
\frac{1}{\Omega}=y \text { et } \frac{2 \pi c}{\mathrm{Q}_{\nu}}=\mathrm{K} \\
d \Omega=-\Omega^{2} d y=-\frac{d y}{y^{2}}
\end{array}\right.\right.
$$

d'où :

$$
-x^{2} \frac{d ! y}{y}+\mathrm{K} \cdot \frac{1}{y} d x=2 x d x
$$

c'est-à-dire :

$$
\frac{d y}{d x}+2 \frac{y}{x}=\mathrm{K} \cdot \frac{1}{x^{2}}
$$

La solution de cette équation différentielle nous donne :

$$
y=\frac{\mathrm{K}}{x}+\frac{\mathrm{C}}{x^{2}}
$$

ou bien, si nous employons la notation originale :

$$
\Omega=\frac{\left[\theta+\left(\mathrm{Q}_{1} / \mathrm{Q}_{p}\right) \cdot 2 \pi\right\rceil^{2}}{-\frac{c}{\mathrm{Q}_{p}} \cdot 2 \pi\left\lceil\theta+\left(\mathrm{Q}_{1} / \mathrm{Q}_{p}\right) 2 \pi\right\rceil+\mathrm{C}}
$$

$\mathrm{C}$ étant une constante d'intégration qui se détermine par le choix de l'aire de section de la volute en n'importe quel point particulier.

Pour le cas spécial $\mathrm{Q}_{1}=0, c=v$ et $\mathrm{C}=0$, l'équation (9) s'écrit sous la forme classique suivante :

$$
\Omega=\frac{\left(\mathrm{Q}_{p} / 2 \pi\right) \cdot 0}{v}=\frac{\mathrm{Q}}{v}
$$

La constante $\mathrm{C}$ de l'équation (9) est donnée par :

$$
\mathrm{C}=\frac{\left[\theta+\left(\mathrm{Q}_{1} / \mathrm{Q}_{p}\right) \cdot 2 \pi\right]^{2}}{\mathrm{Q}}-\frac{c}{\mathrm{Q}_{\nu}} \cdot 2 \pi\left[0+\frac{\mathrm{Q}_{1}}{\mathrm{Q}_{p}}\right] 2 \pi
$$

Pour les grandes valeurs de $\Omega$, et pour une valeur donnée de $\theta$, la constante $C$ aura des valeurs très faibles ou même négatives, et le dénominateur de l'équation (9) sera éventuellement zéro ou même négatif pour des faibles valeurs de $A$, ce qui est irréalisable dans la pratique.

Dans un tel cas, si nous substituons à l'entrée de la volute une petite valeur positive de l'aire $\Omega$ dans cette partie de la volute, nous obtenons une pression statique relativement faible, ce qui permettra à un débit également plus élevé de s'écouler de la roue; d'autre part, la pression statique à l'autre extrémité de la volute sera plus élevée, et le débit sortant de la roue en cet endroit sera plutôt plus faible. La définition du rayon moyen $\mathrm{R}_{a v}$ et de la composante tangen. tielle de la vitesse absolue $(c)$ au rayon moyen est donnée dans la suite du texte.

On voit d'après la figure 1 que toute l'aire de section de la volute est considérée comme étant la somme de deux sections désignées dans le présent mémoire :

$$
\begin{aligned}
& \text { - la section de la volute } \Omega \text {, el } \\
& \text {-. la section du jet } \Omega_{j} \text {. }
\end{aligned}
$$

Il est supposé que les répartitions des vitesses dans ces deux sections sont indépendantes l'une de l'autre, et que dans les deux cas le débit suit la loi de la quantité de mouvement angulaire :

$$
\mathrm{R} \cdot \mathrm{V}=\mathrm{C}^{\text {te }}
$$


A mesure que le transfert de quantité de mouvement se produit entre l'intérieur et l'extérieur de la volute (de $R_{1}$ en $R_{2}$, voir fig. 1), la composante tangentielle de la vitesse variera de telle façon que :

$$
\mathrm{R} . \mathrm{V}=\mathrm{C}^{\mathrm{te}}=\mathrm{R}_{\mathrm{I}} \mathrm{V}_{t}
$$

Lorsque $R=R_{1}$, la composante tangentielle de la vitesse absolue $\left(V_{t}\right)$ définira la constante:

$$
\mathrm{C}^{\mathrm{tc}}=-\mathrm{R}_{1} \times \mathrm{V}_{t}
$$

Supposons maintenant que le transfert de quantité de mouvement du jet au débit de la volute soit concentré en un seul point $R_{a r}$, la valeur de la composante langentielle de la vitesse absolue du jet en ce point ctant $c$, nous aurons alors :

$$
\mathrm{R}_{1} \mathrm{~V}_{t}=\mathrm{R}_{u t} \cdot c
$$

Etant donné que $Q$ est le débit dans une section de la volute (fig. 3) :

$$
\mathrm{Q}=\int_{\mathrm{R}_{1}}^{\mathrm{I} \Omega} \int_{y_{\mathrm{m} \text { In }}}^{y_{\max }} d y d x \cdot V
$$

et pour la définition de la vitesse moyenne:

$$
V=\frac{Q}{Q}
$$

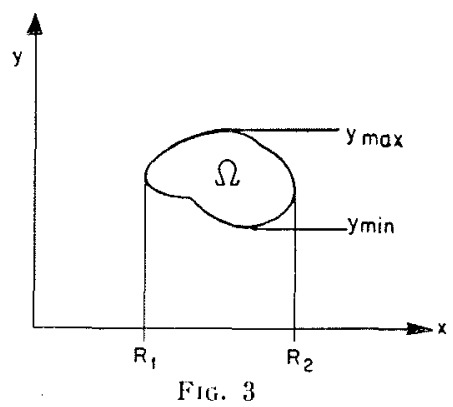

Si nous admettons que lorsque $\mathrm{R}=\mathrm{R}_{\text {an }}$ la valeur de la vitesse $\left(V_{0}\right)$ dans la volute est égale à la vitesse moyenne, la condition pour la quantité de mouvement angulaire sera:

$$
\mathrm{V}_{1} \mathrm{R}_{1}=\mathrm{V}_{2} \mathrm{R}_{2}=\mathrm{V} \cdot \mathrm{R}_{a v}=\mathrm{X} \cdot \mathrm{V}_{x}=\mathrm{C}_{1}=\mathrm{C}^{\mathrm{te}}
$$

Nous tirons des équations (14) et (16) :

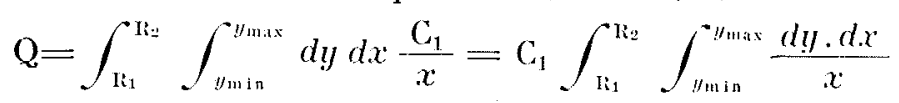

Avec (15) et (16):

$$
\begin{aligned}
& \mathrm{Q}=\mathrm{QV}=\mathrm{VR}_{u v} \int_{\mathrm{R}_{1}}^{\mathrm{R}: 2} \int_{y_{\mathrm{m} \text { in }}}^{y_{\max }} \frac{d y \cdot d x}{x}
\end{aligned}
$$

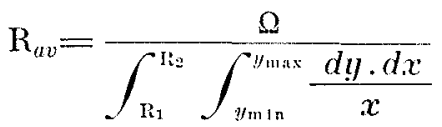

Lorsque nous avons établi l'équation (9), nous avons admis que le débit d'eau traversant la section ABCD (figs. 2) était égal à celui fourni par la roue, soit $d q$.

Toutefois, en réalite, un petit jel partant de la roue au point $A$ (lig. 4) continuera dans le

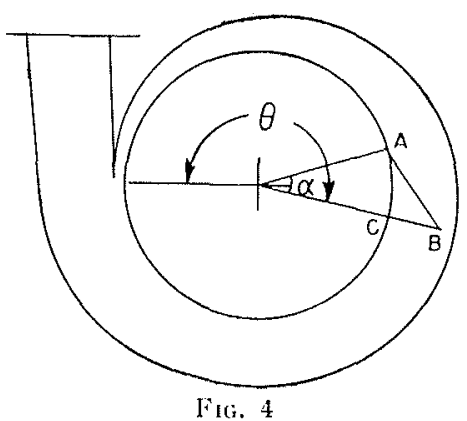

sens de la vitesse absolue el s'ajoutera au débit de la volute entre $A$ et $B$. Le point $B$ est considéré comme celui oi le jet s'est mélangé en totalité au débit dans la volute.

Rappelons que l'aire de section de la volute en un point quelconque se divise en $\Omega$, section occupée par un écoulement lranquille, et en $Q_{j}$, zone où le jet persiste.

Considérant la section de la volute en BC, nous voyons que l'écoulement à cet endroit se compose de :

1. La totalité du débit sorlant de la rone, de la languetle au point $A$.

2. Un certain pourcentage du débit sortant entre $A$ et $\mathrm{C}$.

Le débit sortant entre $A$ et $C$ est $\left(Q_{p} / 2 \pi\right) \propto$. Le débit sortant de la roue entre $A$ et $C$ qui n'est transféré à la volute qu'après la section BC, est:

$$
\frac{\mathrm{Q}_{n}}{2 \pi} \cdot \frac{\alpha}{a}
$$

« $a »$ étant un coefficient dont la valeur varie en fonction de la forme de la volute et surtout de la distance entre la languette et la section considérée.

Considérant l'équation (19), nous obtenons pour le débil dans l'aire de section de la volute a la section $\mathrm{CB}$ :

$$
Q^{\prime}=\frac{Q_{\prime \prime}}{2 \pi} \cdot 0+Q_{1}-\frac{Q_{y}}{2 \pi} \cdot \frac{\alpha}{a}
$$

Dans le cas où le jet persiste jusqu'a la paroi extérieure de la volute, la position de cette paroi déterminera la valeur de a dans l'équation (18), et lorsque, dans ce cas, le jet aura alteint la paroi extérieure de la volute, nous pourons admettre que $\alpha$ est donné approximativement par :

$$
\alpha=\alpha_{1}+k \theta
$$


$\alpha_{1}$ étant la valeur de $\approx$ lorsque $\theta=0$, et $k$ étant un coefficient qui sera déterminé par le choix du profil extérieur de la volute.

Il est nécessaire, pour déterminer la valeur de $a$, de disposer de données complémentaires sur les caractéristiques des jets sortant de la roue; si on divise la volute en plusieurs parties pour le calcul, ceci permettra d'accepter une valeur constante de a pour chacune de ces parties.

Si nous substituons: $\quad-\frac{Q_{n}}{2 \pi}=A$

l'équation (6) aura la forme : $\frac{Q^{2}}{\Omega}+A . c . d \theta=\frac{(Q+A d \theta)^{2}}{\Omega+d Q}$

Employant alors les équations (19) et (20) ci-dessus :

et substituant :

$$
\mathrm{Q}=\mathrm{A} 0+\mathrm{Q}_{1}-\mathrm{A}\left(\frac{\alpha_{1}}{a}+\frac{k}{a} \theta\right)
$$

$$
\left\{\begin{array}{c}
\mathrm{A}[1-(k / a)]=\mathrm{L} \\
\mathrm{Q}_{1}-\mathrm{A}\left(\alpha_{1} / a\right)=\mathrm{M} \\
\mathrm{Q}=\mathrm{L} \theta+\mathrm{M}
\end{array}\right.
$$

Nous tirons des équations (21) et (22):

en substituant :

$$
\frac{(\mathrm{L} 0+\mathrm{M})^{2}}{\Omega}+\mathrm{A} \cdot c \cdot d \theta=\frac{(\mathrm{L} 0+\mathrm{M}+\mathrm{Ad} d \theta)^{2}}{\Omega+d \Omega}
$$

$$
\left\{\begin{array}{rr}
\frac{\mathrm{M}}{\mathrm{L}}=\mathrm{D} & \mathrm{L}^{2} x^{2} y+\mathrm{A} \cdot c \cdot d x=\frac{\mathrm{L}^{2}\left[x+(\mathrm{A} / \mathrm{L}) d x^{2}{ }^{2}\right.}{(1 / y)-\left(1 / y^{2}\right) d y} \\
0+\mathrm{D}=x & \text { c'est-à-dire : } \\
\frac{1}{\Omega}=y & \mathrm{~L}^{2} x^{2}-\frac{\mathrm{L}^{2} x^{2}}{y} d y+\frac{\mathrm{A} c}{y} d x-\frac{\mathrm{A} c d x d y}{y^{2}} \\
d \theta=d x & -\mathrm{L}^{2} \cdot x^{2}+\mathrm{L}^{2} \cdot 2 x \frac{\mathrm{A}}{\mathrm{L}} d x+\mathrm{L}^{2} \frac{\mathrm{A}^{2}}{\mathrm{~L}^{2}} d x^{2} \\
d y=-\frac{d Q}{\Omega^{2}} &
\end{array}\right.
$$

Négligeant les deuxièmes ordres des petites quantités :

et substituant :

$$
\begin{gathered}
-\frac{d y}{y}+\frac{\mathrm{AC}}{y \mathrm{~L}^{2} x^{2}} d x=2 \frac{\mathrm{A}}{\mathrm{L} x} d x \\
\frac{d y}{d x}+2 \frac{\mathrm{A}}{\mathrm{L}} \frac{y}{x}=\frac{\mathrm{AC}}{\mathrm{L}^{2}} \cdot \frac{1}{x^{2}}
\end{gathered}
$$

$$
\begin{aligned}
\mathrm{F} & =\frac{2 \mathrm{~A}}{\mathrm{~L}}=\frac{2}{1-(k / a)} \\
\mathrm{K}=\frac{\mathrm{A} \cdot c}{\mathrm{~L}^{2}} & =\frac{c}{\mathrm{~A}[1-(k / a)]^{2}} \\
\frac{d y}{d x}+\mathrm{F} \frac{y}{x} & =\mathrm{K} \frac{1}{x^{2}}
\end{aligned}
$$

La solution de l'équation (23) est :

c'est-à-dire :

$$
Q=\frac{(\mathrm{F}-1) x^{2}}{\mathrm{~K} x+\mathrm{C}(\mathrm{F}-1)}=\frac{\left[\frac{2}{1-(k / a)}-1\right]\left[\theta+\left.(\mathrm{M} / \mathrm{L})\right|^{2}\right.}{\mathrm{c} / \mathrm{A} \mid 1-(k / a)]^{2}[0+(\mathrm{M} / \mathrm{L})]+\mathrm{C}\left[\frac{2}{1-(k / a)}-1\right]}
$$

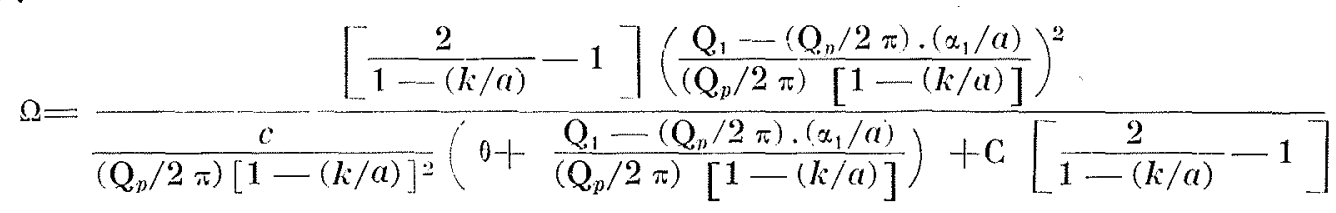

Dans le cas où $\alpha_{1}=0$ et $k=0$, l'équation (24) devient identique à l'équation (9). 
Dans l'équation (24):

$k$, Coefficient dont la valeur est fonction de la courbure extérieure de la volute.

a, Variable dont la valeur croît avec 0 . Puisque nous l'avons supposée constante, il est nécessaire de subdiviser la volute en plusieurs éléments et d'appliquer l'équation (24) successivement dans chaque section, utilisant les valeurs moyennes de $k$ et $a$ correspondant à chacun de ces éléments.

II est nécessaire, si l'on divise la volute en plusieurs éléments, de commencer les calculs à partir de sa petite extrémité et de déterminer le débit $Q$ à la fin de l'élément en question; ce $Q$ devient alors $Q_{1}$ pour l'élément suivant.

$\alpha_{1}$. Cette valeur se détermine une fois que l'on a défini la courbure extérieure et que l'on connait le sens de la vitesse absolue à la sortie de la roue. c. Est le facteur le plus important pour déterminer l'aire de la section.

Pour déterminer celle-ci, il est nćcessaire de connaître le sens de la vitesse absolue, qui varie par rapport à l'angle des aubes de la roue en fonction de la valeur de la vitesse radiale.

C. Est la constante de l'équation et se détermine en choisissant une section de la volute en un lieu particulier.

Si les valeurs de cette section sont relativement grandes, on aura des faibles valeurs de $\mathrm{C}$, ou même des valeurs négatives, et les résultats des calculs n'auront aucune valeur pratique. Tout départ de la forme calculée entrainera une réparlition non uniforme des pressions statiques autour de la périphérie de la volute. Dans le cas où la pression statique à l'entrée de la volute est égale à celle existant à sa sortie, il se produira généralement une baisse de la pression statique sur la partie intermédiaire.

\section{RẼSULTÁTS DE LA PREMIËE EXPÉRIENCE SUR UNE VOLUTE DE FORME CLASSIQUE}

\section{Description de la pompe utilisée pour les essais}

Diamètre extérieur de la roue : $91 / 2$ pouces. Vitesse d'essai : $1.350 \mathrm{tr} / \mathrm{mn}$.

Largeur à la sortie de la roue : 0,55 pouce. Nombre d'aubes : 8.

Angle des aubes à la sortie : $24^{\circ}$. Pompe à simple aspiration.

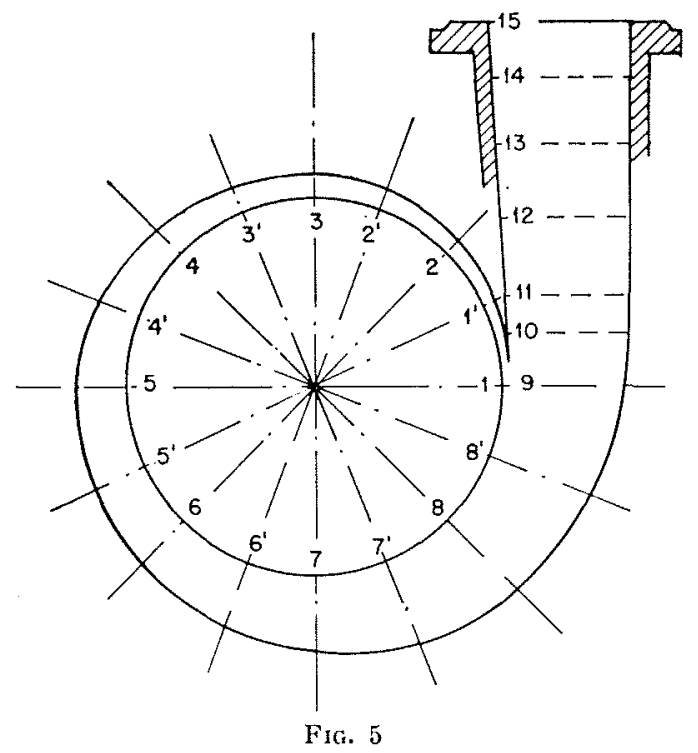

Les 16 points de mesure des pressions disposées autour de la volute sont numérotés dans la figure 5 .

Nous avons installé un anneau séparant la volute de l'espace entre la roue et le corps de pompe, ceci afin d'éviter toute circulation d'eau entre la roue et le corps de pompe (Fig. 6).

Le jeu à la languette est d'environ $1 / 16^{\circ}$ de pouce.

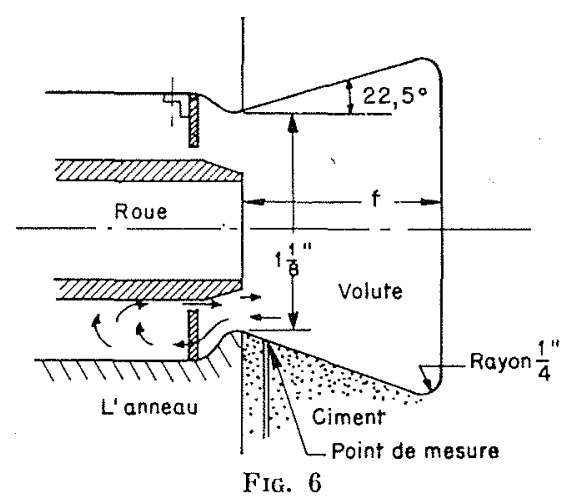

\section{Aires de section de la volute}

Les aires de section et la forme de la volute calculées pour cette expérience sont données dans le tableau I, page 647, et sur la figure 6 . 


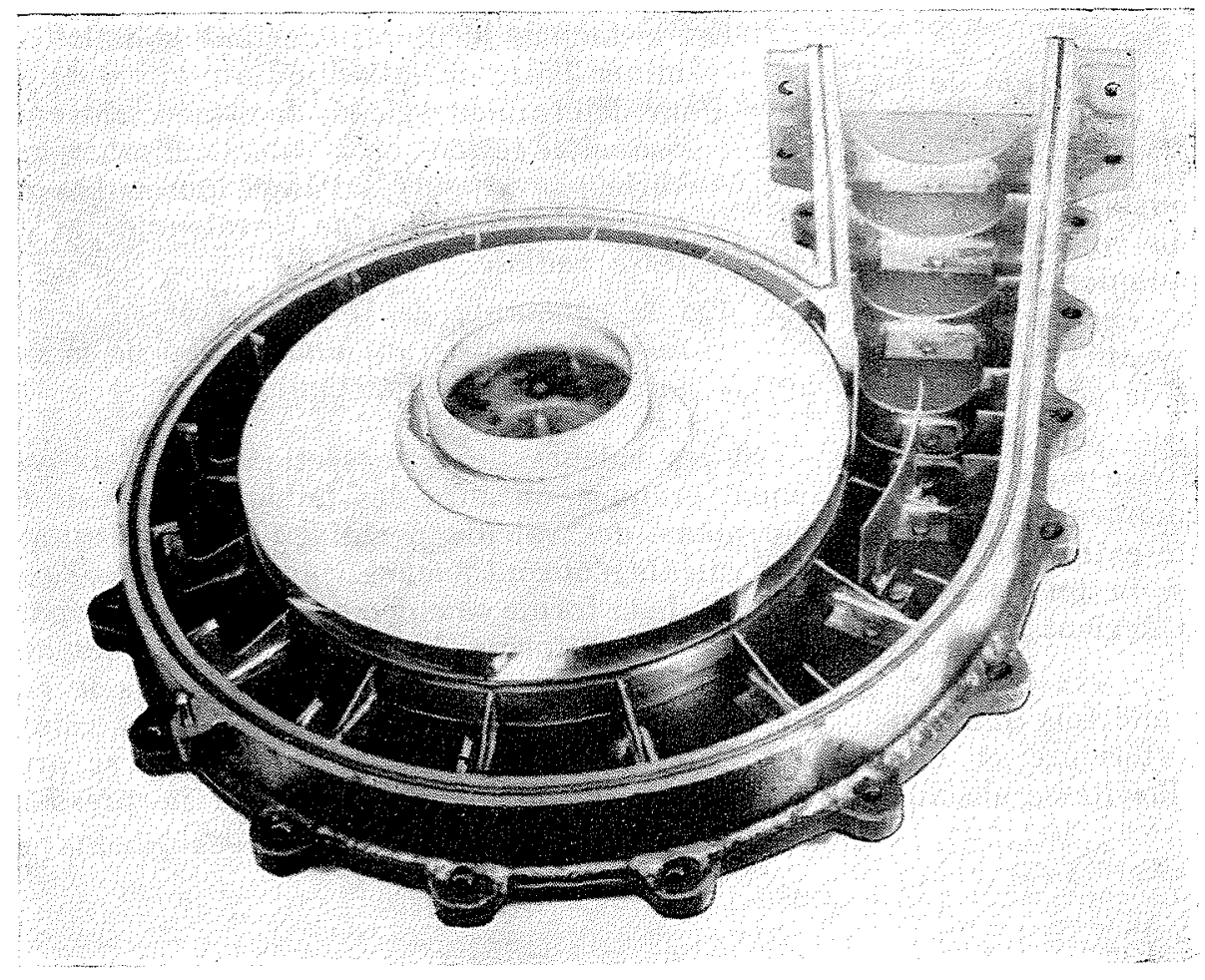

FIG. $7_{1}$

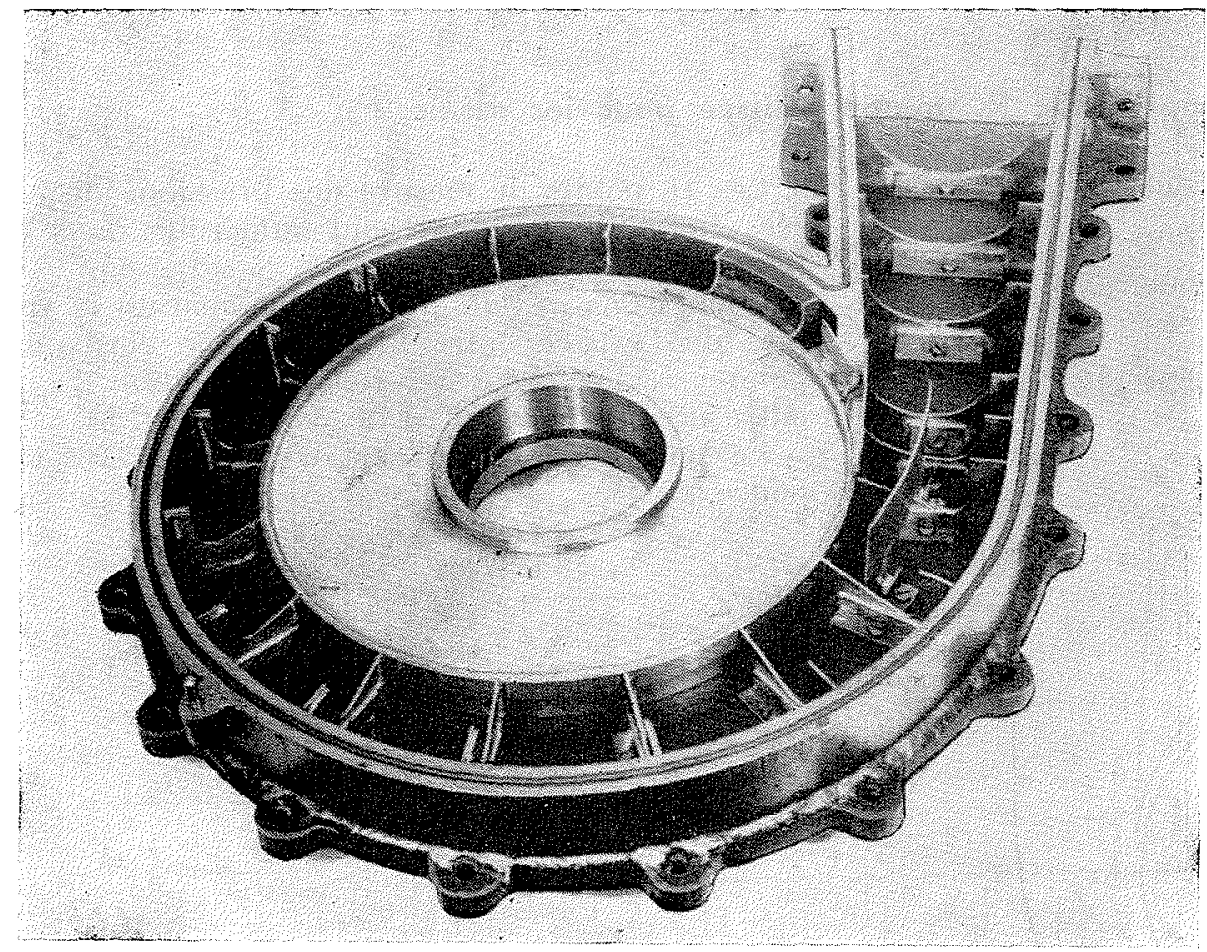

Fig. $7_{1}$ et Fig. $\bar{\gamma}_{2}$

Gabarits utilisés pour déterminer le profil intérieur avant

le remplissage au ciment.

Fili. 7 ,

Ces sections sont elliptiques de lat position $\mathrm{n}^{\circ} 11$ à la position $\mathrm{n}^{0} 15$ (voir fig. 5).

A la section n" 9 (aire : 3,062 pouces carrés), la section diminue d'abord jusqu'à ce que l'on arrive à la section $n^{\circ} 11$ (aire : 2,50 pouces carres). Cette contraction a pour objet d'amé- liorer la répartition des vitesses à travers la section avant que le fluide ne passe à l'expansion entre les positions 11 et 15 .

Nous avons découpé les sections dont les positions sont indiquées sur la figure 5 , dans de la bole de laiton, en les fixant solidement à la vo- 
lute (fig. 7), et en remplissant les espaces entre elles avec un mélange de sable et de ciment. Afin de permettre de modifier plus facilement la forme de la section, nous avons dosé ce mélange dans un rapport de $1 / 10$ du ciment au sable pour la couche de fond, et de $1 / 3$ pour la surface. Finalement, nous avons peint la surface pour la rendre plus lisse. Aucune détérioration du ciment ou de la peinture n'a été observée, ceci après environ une centaine d'heures de fonctionnement.

TaBLEAU I

\begin{tabular}{|c|c|c|}
\hline Position $n^{\circ}$ & $f$ en pouces & Aire en pouces carrés \\
\hline 1 & $0,11 \mathrm{i}$ & 0,103 \\
\hline $1^{\prime}$ & 0,180 & $0, i s \%$ \\
\hline 2 & 0,278 & 0,316 \\
\hline $2^{\prime}$ & 0.378 & 0,455 \\
\hline 3 & 0,482 & $0,606 i$ \\
\hline $3^{\prime}$ & 0,589 & 0,768 \\
\hline 4 & 0,696 & $0,9+1$ \\
\hline $4^{\prime}$ & 0,805 & 1,125 \\
\hline 5 & 0,910 & 1,320 \\
\hline $5^{\prime}$ & $1,(1220$ & 1.521 \\
\hline 6 & 1,126 & 1.728 \\
\hline $6^{\prime}$ & 1,230 & 1,940 \\
\hline 7 & 1,330 & 2,155 \\
\hline $7^{\prime}$ & 1,430 & 2,375 \\
\hline 8 & 1,528 & 2,600 \\
\hline $8^{\prime}$ & 1,626 & 2,829 \\
\hline 9 & 1,718 & 3,062 \\
\hline
\end{tabular}

\begin{tabular}{|c|c|c|c|}
\hline Position n & $\begin{array}{c}\text { Aire } \\
\text { pouces } \\
\text { carrés }\end{array}$ & Grand axe & Petit axe \\
\hline 11 & 2,50 & $2^{\prime \prime}$ & $1,57^{\prime \prime}$ \\
\hline 12 & 3,34 & $2,355^{\prime \prime}$ & $1,85^{\prime \prime}$ \\
\hline 13 & 4,40 & $2,72^{\prime \prime}$ & $2,06^{\prime \prime}$ \\
\hline 14 & 5,70 & $3,105^{\prime \prime}$ & $2,34^{\prime \prime}$ \\
\hline 15 & 7,20 & $3,5^{\prime \prime}$ & $2,62^{\prime \prime}$ \\
\hline
\end{tabular}

\section{Pression statique moyenne}

La définition de la «pression statique moyenne dans la volute » est la moyenne arithmétique de la pression slatique mestrée près de la périphérie de la rone et en des points répartis uniformément autour de celle périphérie. L'emploi de ee teme facilite la comparaison des résultats obtenus dans différentes conditions de fonelionnement.

\section{Répartition de la pression autour de la périphérie}

La figure 8 montre la répartition des pressions statiques autour de la périphérie pour différents

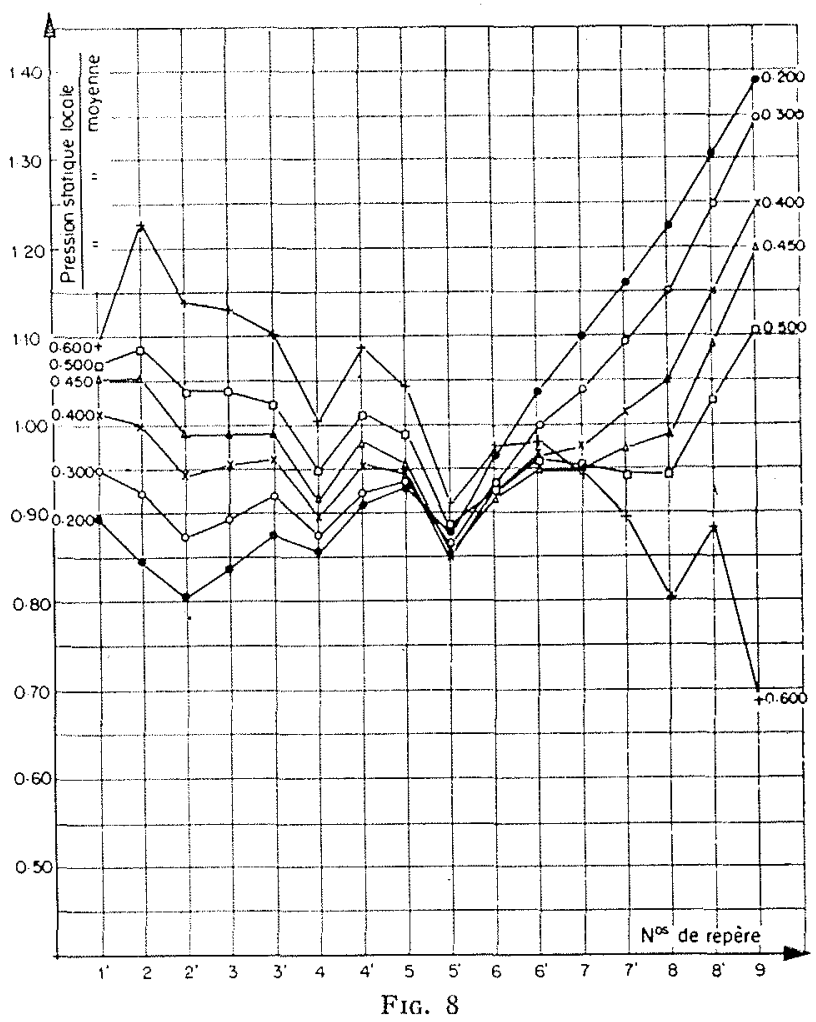

débits. L'ordonnée représente le rapport entre la pression statique locale au point de mesure el la "pression statique moyenne » dans la volute correspondant aux conditions de fonctionnement. Les numéros correspondant aux différentes positions sur la volute (roir fig. 5) sont portés en abscisse. Nous avons fait varier la capacité de la pompe en tant que paramètre pour ces essais, qui ont consisté en six séries effectuées avec les capacités suivantes : 0,$200 ; 0,300$; 0,$400 ; 0,450 ; 0,500 ; 0,600$ pied cube/s. Nous avons relié les valeurs correspondant aux points mesurés par des droites, étant donné qu'il n'a pas été possible de déterminer ce qui se produi- 
sait entre ces points. La répartition la plus uniforme s'est produite pour une capacité légèrement supérieure à $Q=0,520$ pied cube/s, ce qui correspond au rendement maximum (voir fig. 10). La figure 9 montre les mêmes courbes que la figure 8 , mais elles ont été retracées en portant en ordonnè les pressions statiques locales exprimées en pouces de mercure. La constatation la plus intéressante au sujet de ces courbes de répartition des pressions est que, pour des
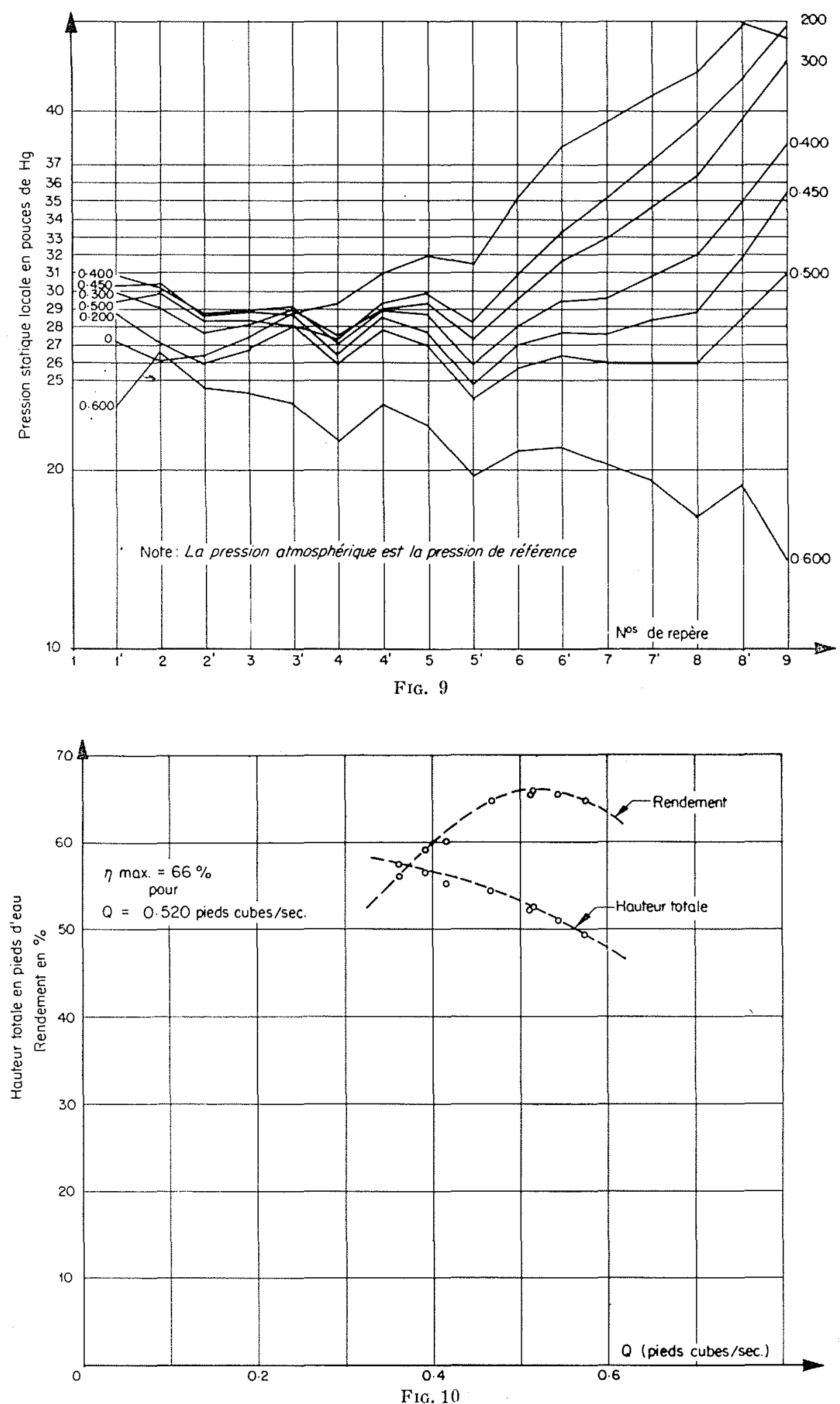
capacités relativement faibles, l'augmentation de pression se produit plus près du côté où se trouve la languette que dans le cas où les débits sont plus élevés. Ceci est vrai jusqu’à la capacité correspondant au rendement maximum. Aux capacités supérieures à celle-ci, il peut se produire une augmentation de pression statique $\dot{a}$ l'entrée de la volute, suivie par une baisse continue de la pression. Etant donné l'insuffisance des points de mesure près de la languette dans cette expérience, et qu'une seule courbe a été obtenue, cette hypothèse n'est pas très bien établie pour les débits plus importants. Toutefois, il est évident que la pression statique sur le côté de la volute où se trouve la languette ne varie pas aussi nettement que du côté de la sortie. Lorsqu'on trace la courbe du rapport de la pression statique à la «pression statique moyenne » telle que nous l'avons tracée dans la figure 8 , les faits apparaissent tout à fait différents : le rapport augmente d'une manièrề continue du côté «languette», tandis qu'il diminue du côté «sortie $\gg$ de la volute : il est constant dans la position $5^{\prime}$ quelle que soit la capacité (fig. 8). La raison n'en est pas évidente, mais pourrait éventuellement être liée à la forme particulière de cette volute.

\section{Résultats de la deuxième expérience}

Le but de cette expérience était d'obtenir certaines données nécessaires pour le calcul de la volute de la troisième expérience.

Bien que nous ayons fait des hypothèses différentes pour le calcul de l'aire de section en n'importe quel endroit et que nous ayons utilisé une forme de section de la volute inhabituelle, pour en faciliter la fabrication, le rendement était aussi bon que celui obtenu au cours d'un essai antérieur avec la même pompe de base et une section de profil classique. Dans ce second essai, la répartition de la pression statique observée autour de la périphérie était beaucoup plus stable et satisfaisante.

Etant donné qu'au cours de cette expérience, nous avons procédé à des mesures de pression statique en 48 différentes sections de la volute, nous avons pu établir l'existence d'une fluctuation de pression distincte superposée à la répartition de pression statique générale.

La volute de la pompe centrifuge utilisée pour cette expérience est calculée d'après l'équation (24).

Nous avons adopté pour la courbure extérieure de la volute une spirale logarithmique et les valeurs de la distance « $f$ (voir fig. 11) sont les suivantes :

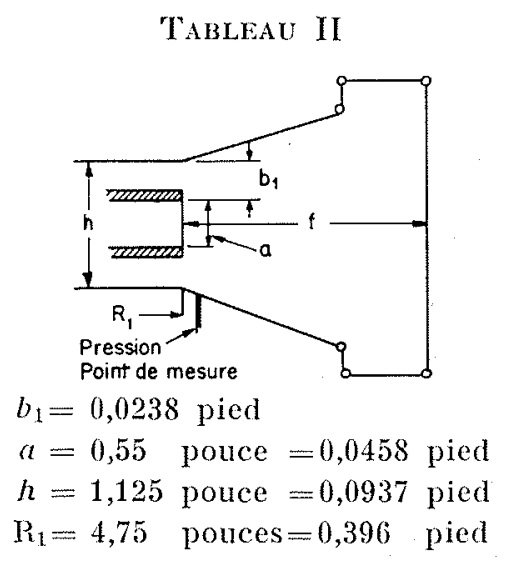

\begin{tabular}{|c|c|}
\hline Position $n^{\circ}$ & $f$ en pouces \\
\hline 9 & $1,718^{\prime \prime}$ \\
\hline $8^{\prime}$ & $1,\left(6^{\prime}\right) 4^{\prime \prime}$ \\
\hline 8 & $1,529^{\prime \prime}$ \\
\hline $7^{\prime}$ & $1,438^{\prime \prime}$ \\
\hline 7 & $1,339^{\prime \prime}$ \\
\hline $6^{\prime}$ & $1,244^{\prime \prime}$ \\
\hline 6 & $1,149^{\prime \prime}$ \\
\hline $5^{\prime}$ & 1,054 \\
\hline 5 & 0,959 \\
\hline $4^{\prime}$ & 0,864 \\
\hline 4 & $0,769^{\prime \prime}$ \\
\hline $3^{\prime}$ & $0,675^{\prime \prime}$ \\
\hline 3 & $0,580^{\prime \prime}$ \\
\hline $2^{\prime}$ & 0,485 \\
\hline 2 & $0,390^{\prime \prime}$ \\
\hline $1^{\prime}$ & 0,295 \\
\hline 1 & $0,200^{\prime \prime}$ \\
\hline
\end{tabular}

Le jeu entre la volute et la roue, mesuré à la languette, est de 0,20 pouce.

La forme de section de la volute est choisie comme indiqué sur la figure 11 , pour permettre une fabrication plus facile sans tenir compte du rendement, et les coins des sections transversales ont été reliés avec du fil de fer pour obtenir une plus grande précision au cours du remplissage des espaces entre les sections transversales calculées. Celles-ci sont découpées dans de la feuille de laiton et fixées solidement au corps de pompe. L'espace qui les sépare est comblé avec un mélange de ciment et de sable dosé à $1 / 6$, et la 


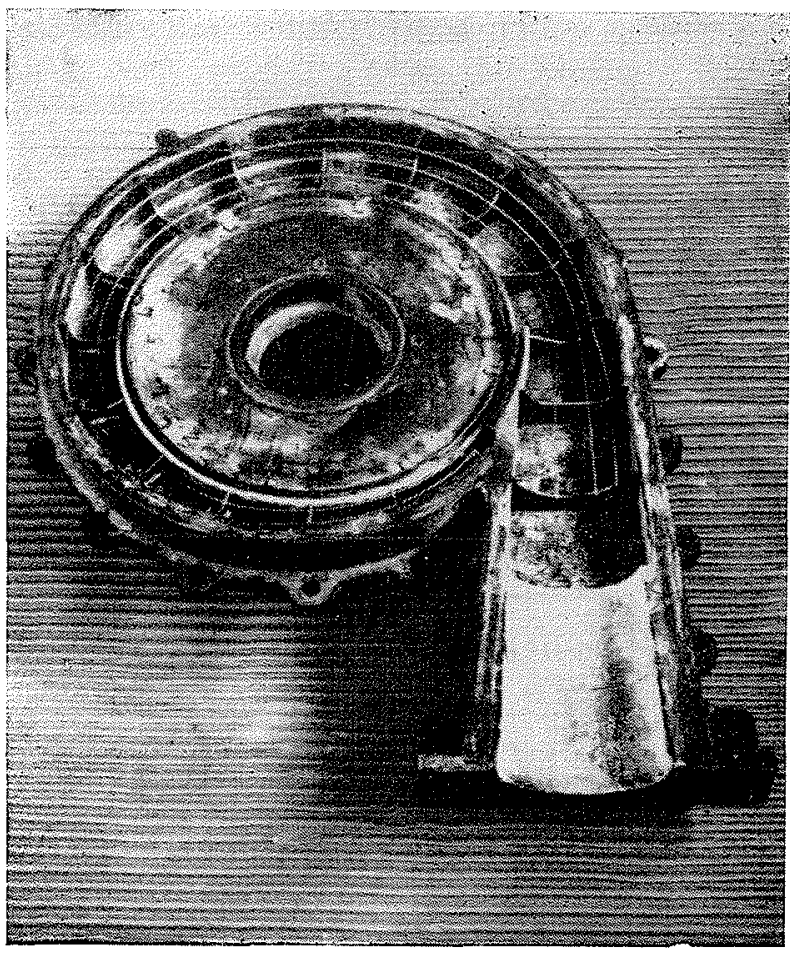

Fic. $11 a$

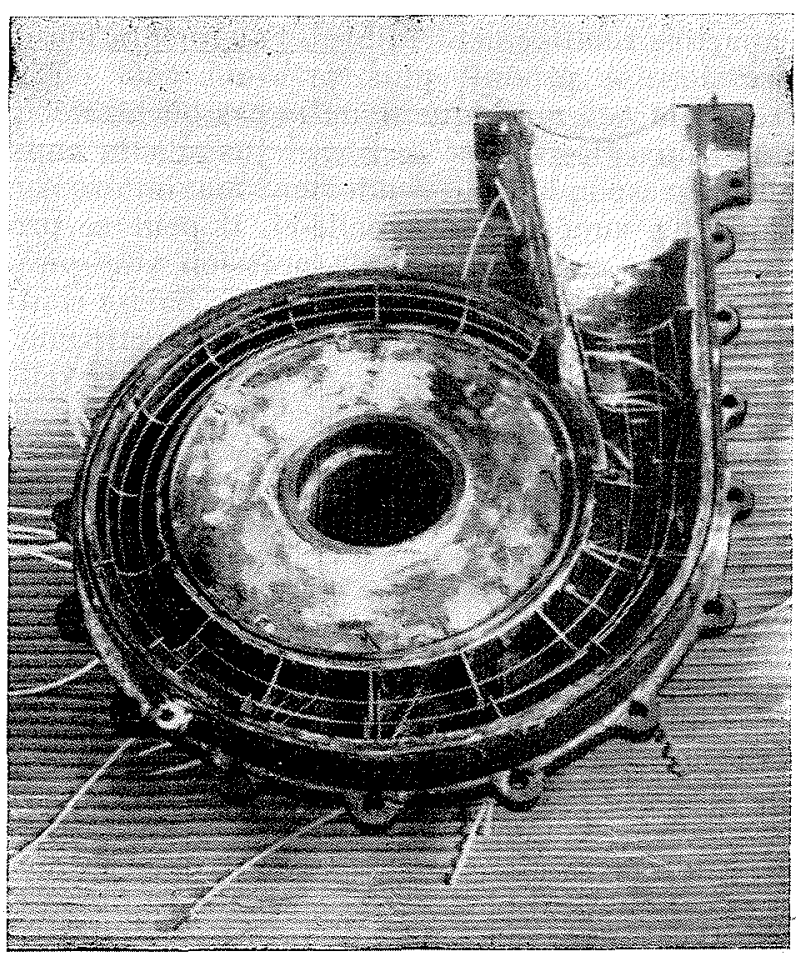

Fia. $11 b$

Fig. 11

Volute comportant les gabarits de section et fils de fer arant le remplissage au ciment.

surface est renforcée avec du ciment pur et peinte de manière à obtenir une surface lisse.

1. - Dans cette expérience, nous cherchons à trouver l'effet d'une augmentation brusque de l'aire de section transversale de la volute, et la répartition de la pression statique autour d'elle.

2. - En certains endroits autour de la périphérie de la volute, nous avons procédé à des mesures en des points très proches les uns des autres, afin d'examiner la continuité de la répartition des pressions statiques autour de la périphérie, ce qui était douteux dans la première expérience.

3. - Nous cherchons à recueillir quelques données, spécialement en ce qui concerne l'écoulement à la sortie de la roue pour une nouvelle conception de la volute, qui suivra.

\section{Calcul de la volute}

Afin de faciliter les essais, la volute a été concue en deux sections différentes avec des capacités en eau différentes dans chacme d'elles, ce qui donne:

1. De la position 1 à la position 4 (fig. 5), une capacité de $Q=0,600$ pied cube $/ \mathrm{s}$.

2. De la position 5 à la position 9, une capacité de $Q=0,300$ pied cube $/ \mathrm{s}$.
Nous avons basé cette étude entre ces deux sections (de la position 4 à la position 5) sur une capacité moyenne de $\mathrm{Q}=0,450 \mathrm{pied}$ cube/s.

Pour la première section de la volute, où nous avons pris une capacité de $Q=0,600 \mathrm{pied}$ cube/s, l'angle de sortie de l'eau venant de la roue par rapport à la tangente, est supposé être de $14^{\circ} 15^{\prime}$ au lieu de $24^{\circ}$, angle de l'aube. Dans ce cas, la composante tangentielle de la vitesse absolue devient :

$$
V_{t}=35 \text { pieds/s. }
$$

Pour la dernière section de la volute, où nous avons pris une capacité, $Q=0,300 \mathrm{pied}$ cube $/ \mathrm{s}$, l'angle de sortie est supposé ètre de $11^{\circ} 20^{\prime}$, ce qui correspond à une composante tangentielle de la vitesse absolue $V_{t}=43$ pieds/s. Si nous supposons égaux l'angle de sortie et l'angle de l'aube, la composante tangentielle serait:

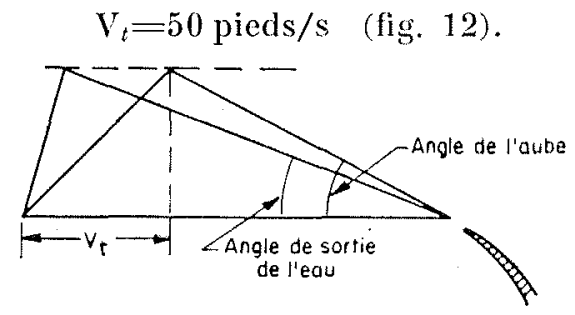

FIG. 12 


\section{Valeur de $Q_{1}$ à la languette}

Le débit pénétrant dans la volute par le jeu à la languette quitte la roue à cette section de la volute où nous avons supposé que la capacité est $Q=0,300$ pied cube par seconde (avant la position 9). On peut évaluer le débit d'eau après avoir fixé les conditions de vitesse à la sortie de la route.

Pour cetie expérience, compte tenu des hypothèses faites an paragraphe ci-dessus, $Q_{1}$ est estimé être le débit d'eau qui sort de la roue le long d'un arc $\pi / 18$ avant la position 9, et entre dans la volute par le jeu à la languette.

Dans ce cas, la valeur de $Q_{1}$ sera :

$$
\mathrm{Q}_{1}=\frac{\pi}{18} \times \frac{0,300}{2 \pi}=0,00835 \text { pied cube } / \mathrm{s}
$$

De même le débit d'eau à la position 9 sera :

$$
\begin{aligned}
\mathrm{Q}=\mathrm{Q}_{1}+\frac{\mathrm{Q}_{0.37}}{2 \pi} \cdot 0_{4}+\frac{\mathrm{Q}_{0.4 \pi}}{2 \pi} \cdot \frac{\pi}{4} & +\frac{\mathrm{Q}_{0.300}}{2 \pi} 0_{5 \text { a } 9} \\
& -\frac{\mathrm{Q}_{0.300}}{2 \pi} \cdot \frac{\alpha_{9}}{a_{0}}
\end{aligned}
$$

oì $\alpha_{9}$ est l'angle correspondant aux points oì un petit jet d'eau est sorti de la roue et a atteint la courbure extérieure de la volute à la position 9 .

$a_{9}$ est un coefficient caractérisant les conditions de mélange de l'écoulement. du jet avec l'écoulement de la volute à l'extrémité de la volute.

Nous pouvons écrire l'équation (24) sous la forme :

$$
\Omega=\frac{A(\theta+B)^{2}}{D(0+B)+A C}
$$

où :

$$
\begin{aligned}
\mathrm{A} & =\frac{2}{1-(k / a)}-1 \\
\mathrm{~B} & =\frac{Q_{1}-\left(\mathrm{Q}_{p} / 2 \pi\right)\left(\alpha_{1} / a\right)}{\mathrm{Q}_{p} / 2 \pi[1-(k / a)]} \\
\mathrm{D} & =\frac{c \cdot 2 \pi}{[1-(k / a)]^{2}}
\end{aligned}
$$

Choisissons les valeurs suivantes du coefficient $« a »$ pour les différentes sections:

$$
\begin{array}{ccrr}
a_{3}=2 & a_{8}=1,875 & a_{7}=1,750 & a_{6}=1,625 \\
a_{5}=1,500 & a_{4}=1,375 & a_{3}=1,250 & a_{2}=1,125 \\
a_{1}=1000 &
\end{array}
$$

\section{Calcul de l'aire de la volute entre les positions 5 et 9}

L'origine de $\theta$ dans l'équation (25) est choisie comme étant la position 4 ' où :

$$
k=\frac{\alpha_{9}-\alpha_{4}}{\pi+(1 / 4) \pi}=0,068
$$

(Les valeurs de $\alpha_{9}$ et $\alpha_{4}$ sont trouvées par détermination géométrique.)

$$
\begin{gathered}
\mathrm{A}=\frac{2}{1-(k / a)}-1=\frac{2}{1-(0,068 / 1,875)}-1=1.065 \\
\mathrm{~B}=\frac{\mathrm{Q}_{1}-\left(\mathrm{Q}_{p} / 2 \pi\right)\left(\alpha_{1} / a\right)}{\mathrm{Q}_{p} / 2 \pi[1-(k / a)]}=\frac{[0,236-(0,300 / 2 \pi) \cdot(\pi / 8) / 1.875]}{0,300 / 2 \pi[1-(0,068 / 1,875)]}=5,0
\end{gathered}
$$

où l'on prend $Q_{1}$ comme étant le débit d'eau dans la volute à la position $4^{\prime}$ où $\theta=0$.

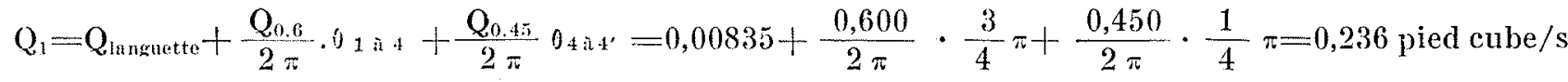

$$
\begin{aligned}
& \mathrm{D}=\frac{c .2 \pi}{\mathrm{Q}_{p}(1-k / a)^{2}}=\frac{37,1 \times 2 \pi}{0,300[1-(0,068 / 1,875)]^{2}}=838
\end{aligned}
$$

Pour obtenir la valeur de $c$, nous avons utilisé le rayon moyen à la position 8 .

$$
\begin{gathered}
\mathrm{R}_{a v}=0,458 \text { pied } \\
\mathrm{V}_{t} \cdot \mathrm{R}_{1}=c \cdot \mathrm{R}_{a w} \\
c=\frac{43 \times 0,396}{0,458}=37,1 \text { pieds par } \mathrm{s} .
\end{gathered}
$$

Pour déterminer $C$, nous avons choisi pour l'aire de section à l'extrémité de la volute :

$$
\varrho_{y}=0,01475 \text { pied carré }
$$

où :

$$
\theta=9 / 8 \pi-1,125 \pi
$$




$$
\begin{aligned}
\mathrm{C}=\frac{(0+\mathrm{B})^{2}}{\Omega}- & \frac{\mathrm{I}(0+\mathrm{B})}{\mathrm{A}}=\frac{(1,125 \pi+5)^{2}}{0,01475} \\
& -\frac{838(1,125 \pi+5)}{1,065}=-1690
\end{aligned}
$$

de sorte que l'équation (25) devient :

$$
\Omega=\frac{1,065(0+5)^{2}}{838(0+5)-1690 \times 1,065}
$$

ce qui donne les aires de sections suivantes :

$$
\begin{array}{ll}
\Omega_{9}=0,0147 \text { pied carré } & \Omega_{\tau^{\prime}}=0,0132 \text { pied carré } \\
\Omega_{8}=0,0141 \text { pied carré } & \Omega_{\bar{\tau}}=0,0128 \text { pied carré } \\
\Omega_{8}=0,0136 \text { pied carré } &
\end{array}
$$

Pour les aires de la volute aux positions 6;, 6, 5 ' et 5 , nous avons appliqué de la même manière les valeurs suivantes:

$$
\begin{aligned}
\Omega_{i} & =0,0128 \text { pied carré pour } 0=1,962 \\
k & =0,068 \\
\alpha_{1} & =\alpha_{4}=\pi / 8 \\
a & =a_{0}=1,625 \\
V_{i} & =43 \text { pieds par seconde } \\
\mathrm{R}_{a b} & =\mathrm{R} a{ }_{0}=0,449 \text { pied } \\
c & =\frac{43 \times 0,396}{0,449}=37,9 \text { pieds } / \mathrm{s}
\end{aligned}
$$

Nous avons obtenu les valeurs suivantes :

$$
\mathrm{A}=1,087 \quad \mathrm{~B}=4,92 \quad \mathrm{D}=866 \quad \mathrm{C}=-1800
$$

Dans ce cas, l'équation (25) devient:

$$
\Omega=\frac{\mathrm{A}(\theta+\mathrm{B})^{2}}{\mathrm{D}(0+\mathrm{D})+\mathrm{CA}}=\frac{1,087(0+4,92)^{2}}{866(0+4,92)-1800 \times 1,087}
$$

et nous avons calculé les valeurs suivantes pour les surfaces aux positions 7 à 5 :

$$
\begin{aligned}
& \Omega_{6^{\prime}}=0,0125 \text { pieds carrés } \\
& \Omega_{6}=0,0122 \text { pieds carrés } \\
& \Omega_{5^{\prime}}=0,0119 \text { pieds carrés } \\
& \Omega_{\bar{\pi}}=0,0116 \text { pieds carrés }
\end{aligned}
$$

\section{Calcul de la surface de la volute entre les positions 1 à 4}

Pour l'aire de section de la position 4, nous avons choisi la valeur $\Omega_{4}=0,0080$ :

$$
\begin{array}{rlrl}
\mathrm{Q}_{1}^{\prime} & =0,00835 \text { pied cube } / \mathrm{s} & k=\frac{\alpha_{3}{ }^{\prime}-\alpha_{1}{ }^{\prime}}{\pi / 2}=0,113 \\
\text { Ra's}=0,4211 \text { pied } & \alpha_{1}=0,113 \\
V_{t}=35 \text { pieds } / \mathrm{s} & a_{3}=1,25 \\
c=\frac{35 \times 0,396}{0,4211}=32,95 \text { pieds } / \mathrm{s} .
\end{array}
$$

Nous avons obtenu les valeurs suivantes de la même manière:

$$
\begin{array}{ll}
\mathrm{A}==1,2 & \mathrm{C}=-121 \\
\mathrm{~B}=-0 & \mathrm{D}=416,5
\end{array}
$$

de sorte que l'équation (25) devient:

$$
\Omega=\frac{A \theta^{2}}{\mathrm{D} \theta-121 \mathrm{~A}}=\frac{1,2 \mathrm{O}^{2}}{416,50-145}
$$

Les aires calculées pour les sections de 4 - 3' -3 et 2 'a l'aide de l'équation (27) sont :

$$
\begin{aligned}
& \Omega_{4}=0,0080 \text { pied carré } \\
& \Omega_{3}=0,0069 \text { pied carré } \\
& \Omega_{3}=0,00584 \text { pied carré } \\
& \Omega_{2}=0,00484 \text { pied carré }
\end{aligned}
$$

Nous ayons utilisé une relation géométrique pour les aires de section dans les positions $1^{\prime}-2$ et $4^{\prime}$.

Nous avons admis que le jet occupait une surface rectangulaire atteignant la paroi extérieure de la volute (fig. 13). Il est admis que l'aire complète de la volute est la somme de ces deux aires (section de la volute-section du jet) (fig. 14). Comme la surface du jet diminue après la position 9, la surface totale se trouve resserrée de la position 9 à la position 12 .

\section{Mesures de la pression statique autour de la périphérie de la volute}

Nous avons procédé à des mesures de la pression statique en 48 points autour de la périphérie de la volute et la sortie de la pompe ellemême. Les positions des points de mesure sont indiquées sur la figure 15. En certains endroits de la volute, nous avons effectué les mesures à

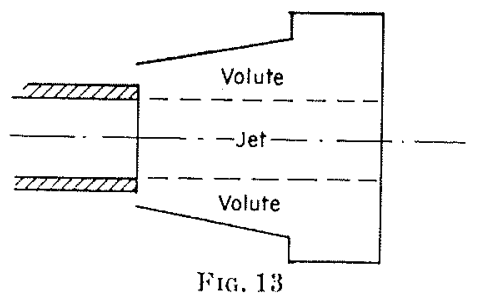

de faibles inlervalles afin d'obtenir une « courbe de répartition des pressions statiques » continue et précise.

La figure 16 montre la forme de la section de la volute ulilisée el la rariation des valeurs $e, g, d$ et $a$. 


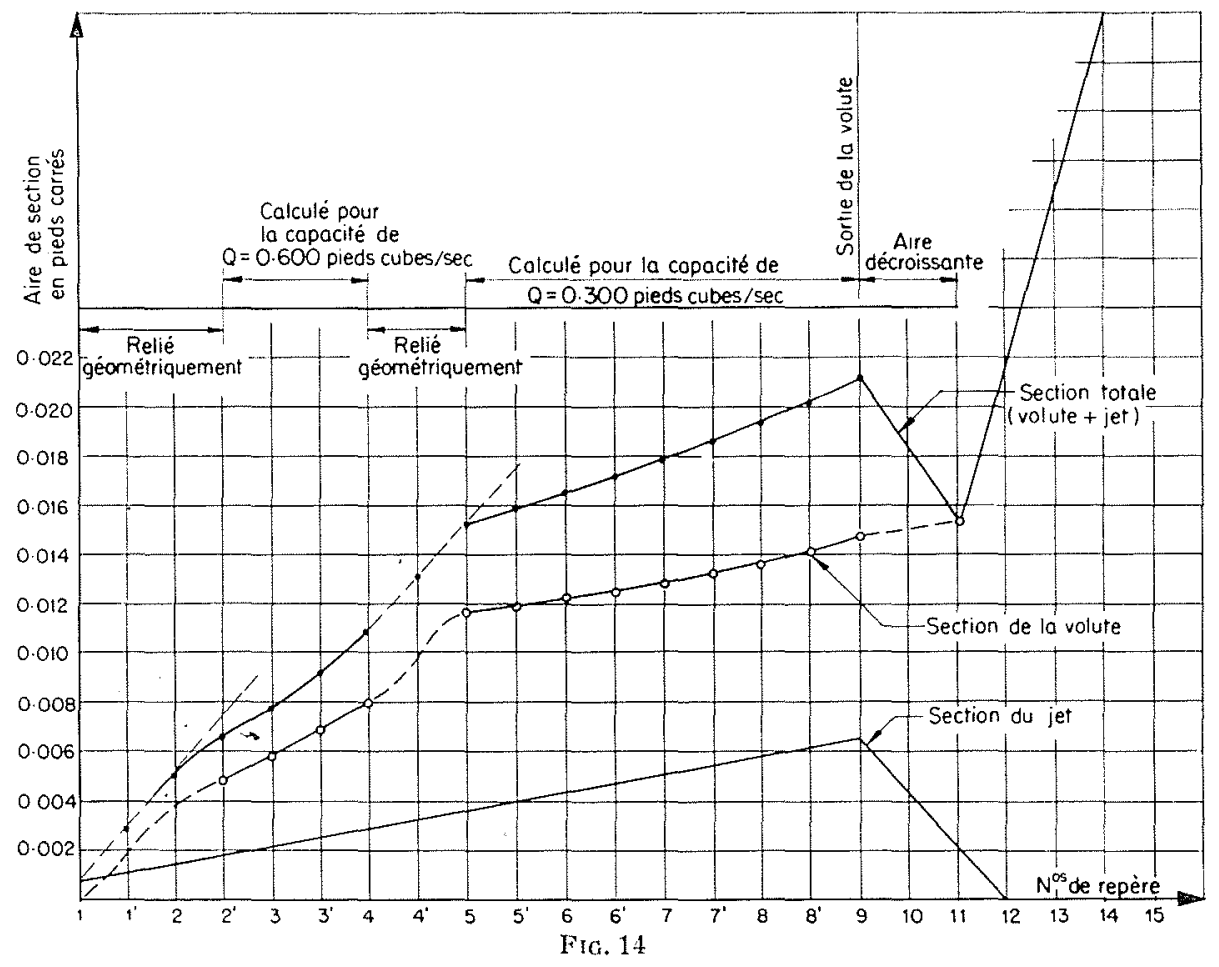

\section{Résultats de l'expérience}

\section{Répartition de la pression statioue}

Les résultats de mesures de pression statique pour 4 capacités sont indiqués sur la figure 17 : $\mathrm{Q}=0,364$ pied cube $/ \mathrm{s} ; \quad \mathrm{Q}=0.430 \mathrm{pied}$ cube $/ \mathrm{s}$; $\mathrm{Q}=0,490$ pied cube $/ \mathrm{s} ; \quad \mathrm{Q}=0,546$ pied cube/s.

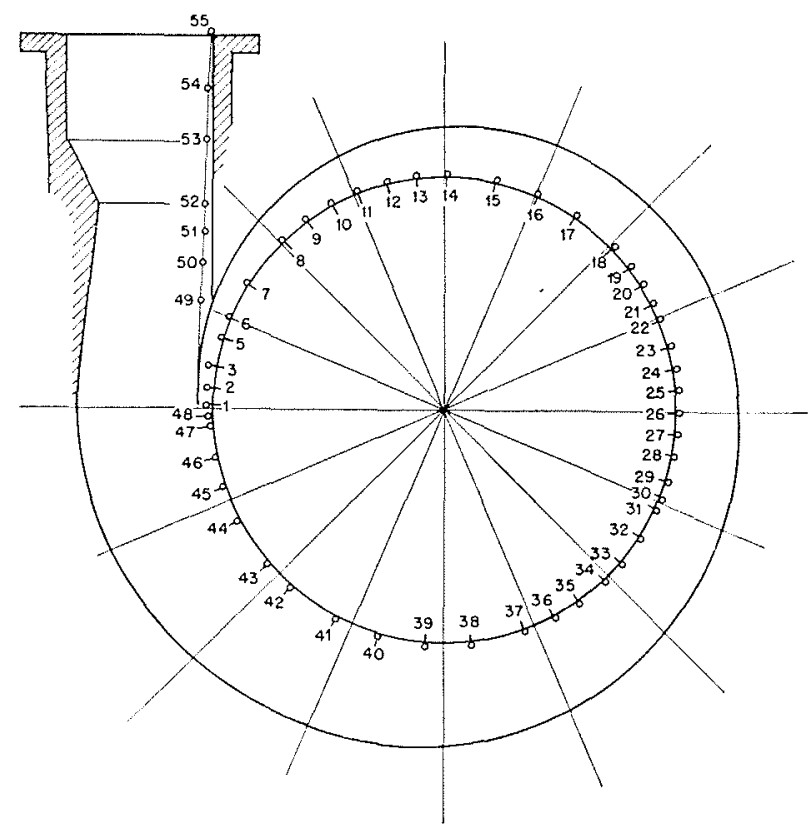

Fia. 13
Les valeurs indiquées sont les rapports des mesures prises avec la pression moyenne à la sortie de la roue.

Les courbes obtenues montrent qu'il existe une fluctuation de la pression statique le long de la courbe générale des pressions. Le caractère de cette fluctuation est beaucoup plus stable que la courbe générale elle-mème lorsque la capacité varie.

Par conséquent, il est vraisemblable que cette fluctuation est due à de faibles divergences, contractions ou dilatations dans la volute, qui se sont produites lors de sa fabrication.

Le second résultat de cette expérience a rapport à la question de l'influence de la forte contraction de l'aire de section entre les positions 9 et 12 (fig. 5).

Bien que la contraction se produise immédiatement après la position 9 (fig. 5) (ce qui correspond au point de mesure 48), la pression statique augmentait jusqu'au point de mesure 49 (fig. 15), où se produisait une brusque chute de pression statique qui se maintenait jusqu'au point de mesure 52.

La conclusion tirée de ce phénomène est que l'effet de la surface du jet sur les calculs ne va pas plus loin que le point de mesure 52, mais que cet effet est plus important entre les points 48 et 49 .

Le rendement maximum obtenu pendant l'essai était $r_{i}=0,66$ pour $Q=0.480$ pied cube $/ \mathrm{s}$, qui est presque le mème que celui obtenu avec la 


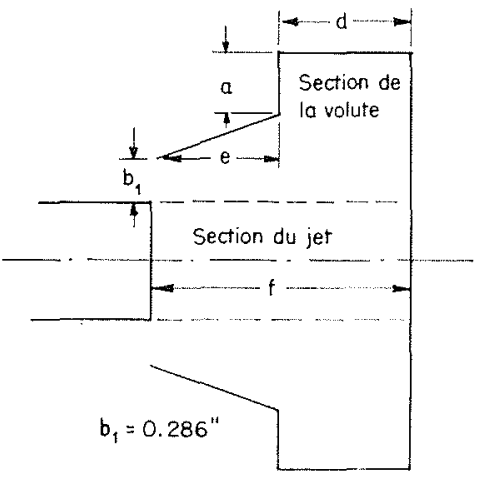

PIti. 16

même pompe avec une volute de conception classique.

Résnltats de la troisieme expérience avec une volute spécialement corrcue pour obtenir une répartition de pression constante autour de la périphérie de la rone.

Le but de cet essai était de déterminer le rendement d'une volute conçue suivant la méthode suggérée. Dans ce qui va suivre, les calculs et les résultats obtenus grace à cet essai sont décrits en détail.

Pour le calcul, quelques données qui devaient être choisies, telles que $V_{l}$, la composante tangentielle de la vitesse absolue a la sorlie de la roue, étaient basées sur les risultats des essais précédents.

Nous avons pris un soin tout particulier au
TABLEAU III

\begin{tabular}{|c|c|c|c|c|}
\hline Position $n^{\circ}$ & $e$ & $g$ & $d$ & $a$ \\
\hline 9 & 0,4 & 0,4 & 1,32 & 0,00 \\
\hline 8 & 0,4 & 0,4 & 1,22 & 0,00 \\
\hline 8 & 0,4 & 0,4 & 1,13 & 0,02 \\
\hline $7^{\prime}$ & 0,4 & 0,4 & 1,04 & 0,04 \\
\hline 7 & 0,4 & 0,4 & 0,94 & 0,095 \\
\hline $6^{\prime}$ & 0,4 & 0,4 & 0,85 & 0,15 \\
\hline 6 & 0,4 & 0,4 & 0,75 & $0, \geq 3$ \\
\hline $5^{\prime}$ & 0,4 & 0,4 & 0,65 & 0,32 \\
\hline 5 & 0,3 & 0,4 & 0,66 & 0,36 \\
\hline $4^{\prime}$ & 0,3 & 0,3 & 0,56 & 0,42 \\
\hline 4 & 0,3 & 0,3 & 0,47 & 0,36 \\
\hline $3^{\prime}$ & 0,3 & 0,4 & 0,37 & 0,25 \\
\hline 3 & 0,2 & 0,4 & 0,38 & 0,17 \\
\hline $2^{\prime}$ & 0,2 & 0,4 & 0,28 & 0,19 \\
\hline 2 & 0,1 & 0,3 & 0,29 & $-0,10$ \\
\hline
\end{tabular}

Les numéros de position dans ce tableau se rapportent il la figure 5.

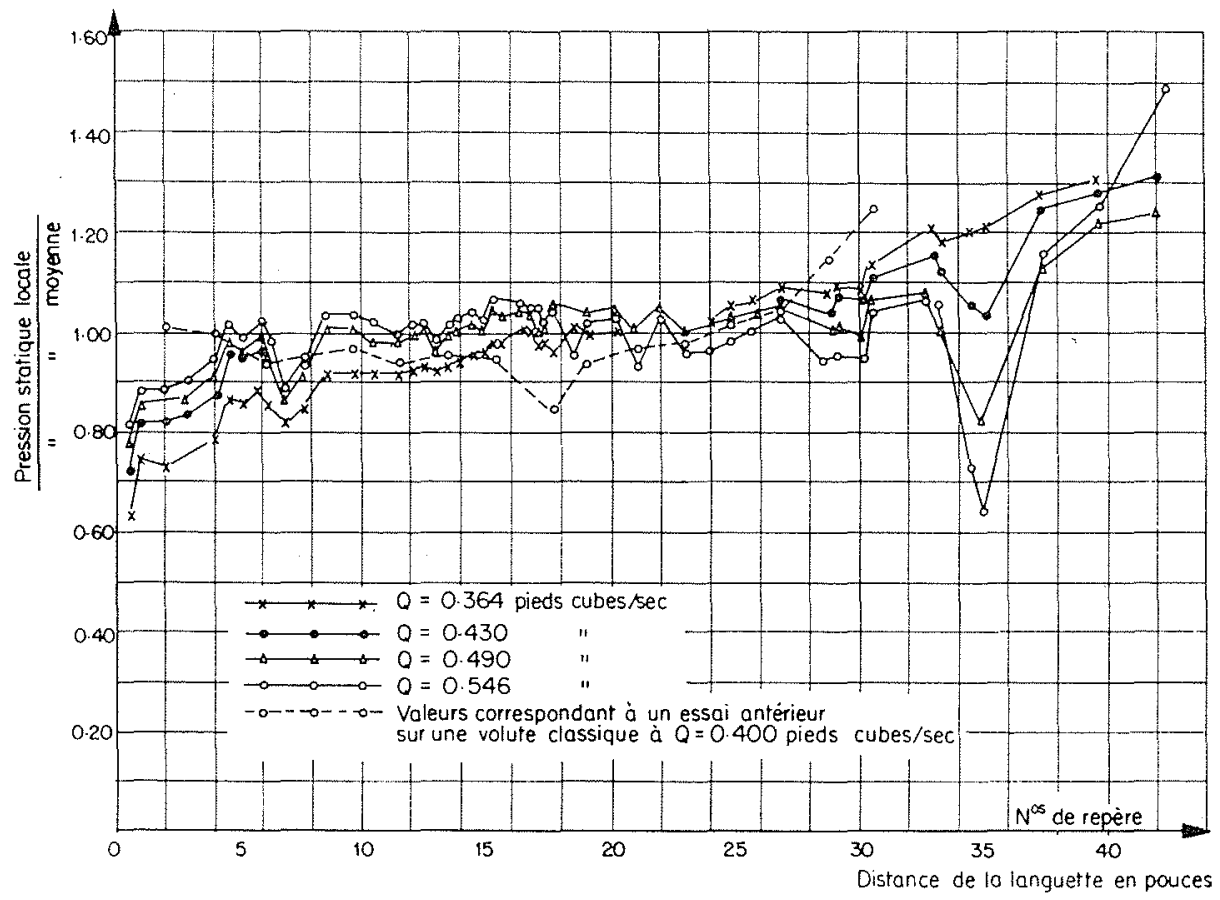

Fis. 17 


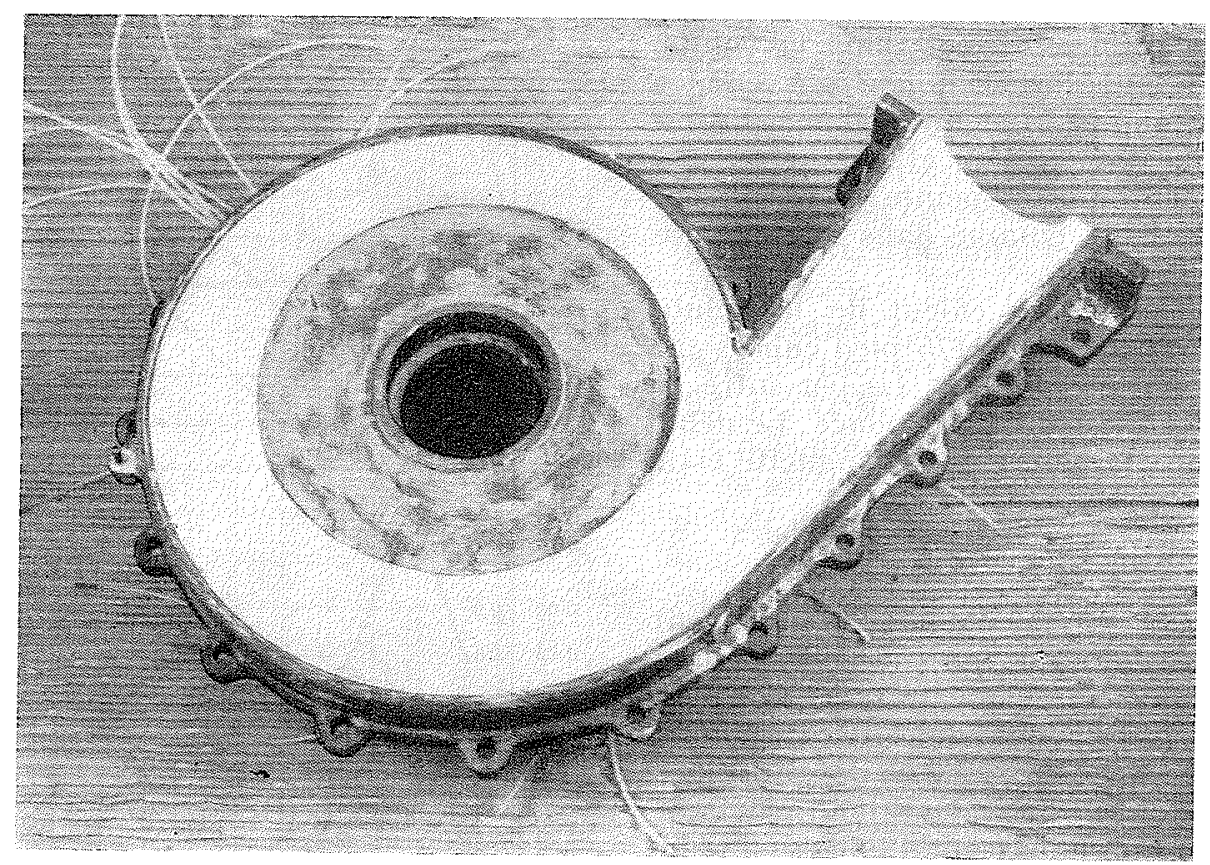

FIG. 18,

Volute remplie de ciment et peinte

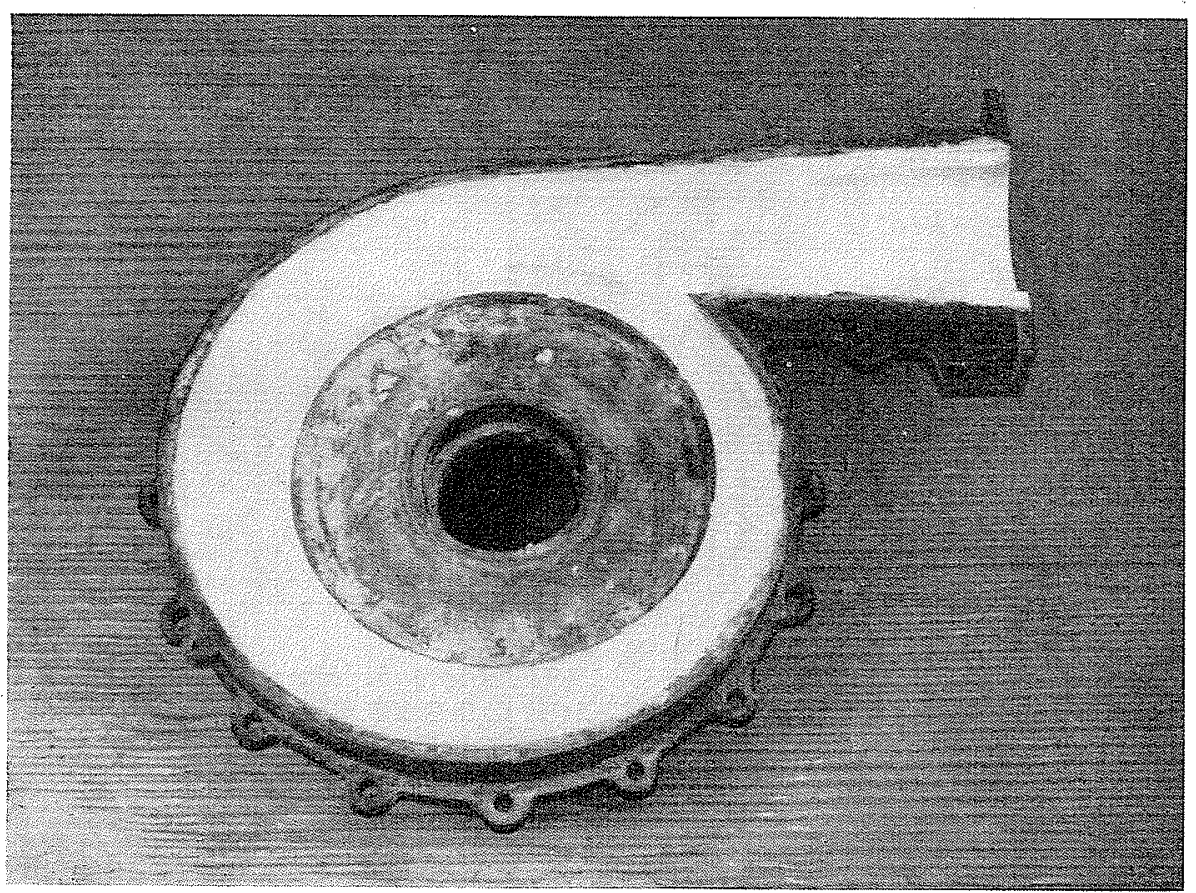

cours de l'expérience à reproduire les conditions des premiers essais sur la même pompe avec une volute de conception classique. Comme toutes les conditions, mise à part la volute, restaient inchangées, il est logique d'attribuer l'augmentation de $2 \%$ dans le rendement de la pompe à la nouvelle conception de la volute. En mème temps, la répartition de la pression statique autour de la périphérie devenait beaucoup plus uniforme.

Les sections transversales calculées en 16 points autour de la volute ont été découpées dans 
de la feuille de laiton et fixées dans la volute.

Les intervalles entre les sections transversales en laiton sont remplis avec un mélange de ciment et de sable dosé à $1 / 6$, et la surface est couverte de ciment pur et peinte pour obtenir une surface plus lisse, comme dans la première expérience (fig. 18).

Nous avons procédé au calcul de la volute pour les valeurs de $Q=0.475$ pied cube $/ \mathrm{s}$ et $\mathrm{N}=1350 \mathrm{tr} / \mathrm{mn}, \mathrm{V}_{t}=42$ pieds $/ \mathrm{s}$. Le jeu entre la languette et la roue est de $=0,2$ pouce. Le débit d'eau entrant dans la volute de la languette est :

$$
\mathrm{Q}_{1}=\frac{\pi}{18} \times \frac{0.475}{2 \pi}=0.0132 \text { pied cube } / \mathrm{s} \text {. }
$$

La valeur $\pi / 18$ a été obtenue d'un profil géométrique très simple, en admettant que $\mathrm{V}_{l}=42$ pieds $/ \mathrm{s}$ pour une capacite $\mathrm{Q}=0,475$ pied cube/s. Comme l'angle de l'aube de la pompe expérimentale est de $24 \%$, si l'eau devait suivre la direction des aubes, la valeur de $\mathrm{V}_{t}$ devrait ètre 46,5 pieds/s. En choisissant 42 pieds/s, pour $V_{t}$, l'angle d'écoulement est de $16,5^{\circ}$ au lieu de $24^{\circ}$, angle de l'aube à la sortie de la roue.

Pour la facilité du calcul, écrivons l'équation (24) sous la forme suivante:

où :

$$
\Omega=\frac{A(0+B)^{2}}{D(0+B)+A C}
$$

$$
\begin{aligned}
& \mathrm{A}=\frac{2}{1-(k / a)}-1 \\
& \mathrm{~B}=\frac{\mathrm{Q}_{1}-\left(\mathrm{Q}_{n} / 2 \pi\right)\left(\alpha_{1} / a\right)}{\mathrm{Q}_{n} / 2 \pi\left[1-\left.(k / a)\right|^{2}\right.} \\
& \mathrm{D}=\frac{c}{\mathrm{Q}_{m} / 2 \pi[1-(k / a)]^{2}}
\end{aligned}
$$

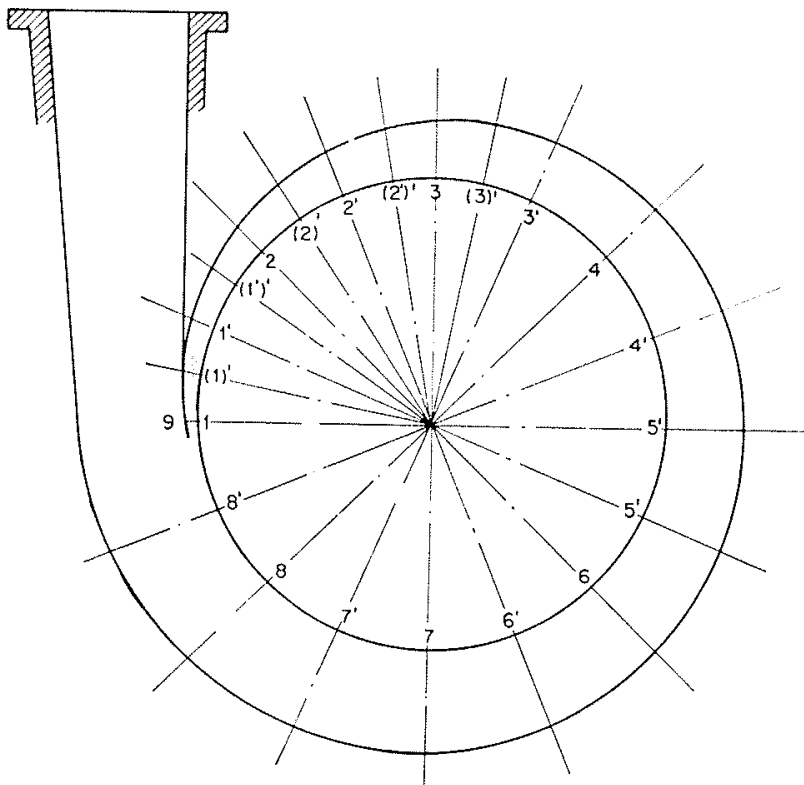

FIG. 19

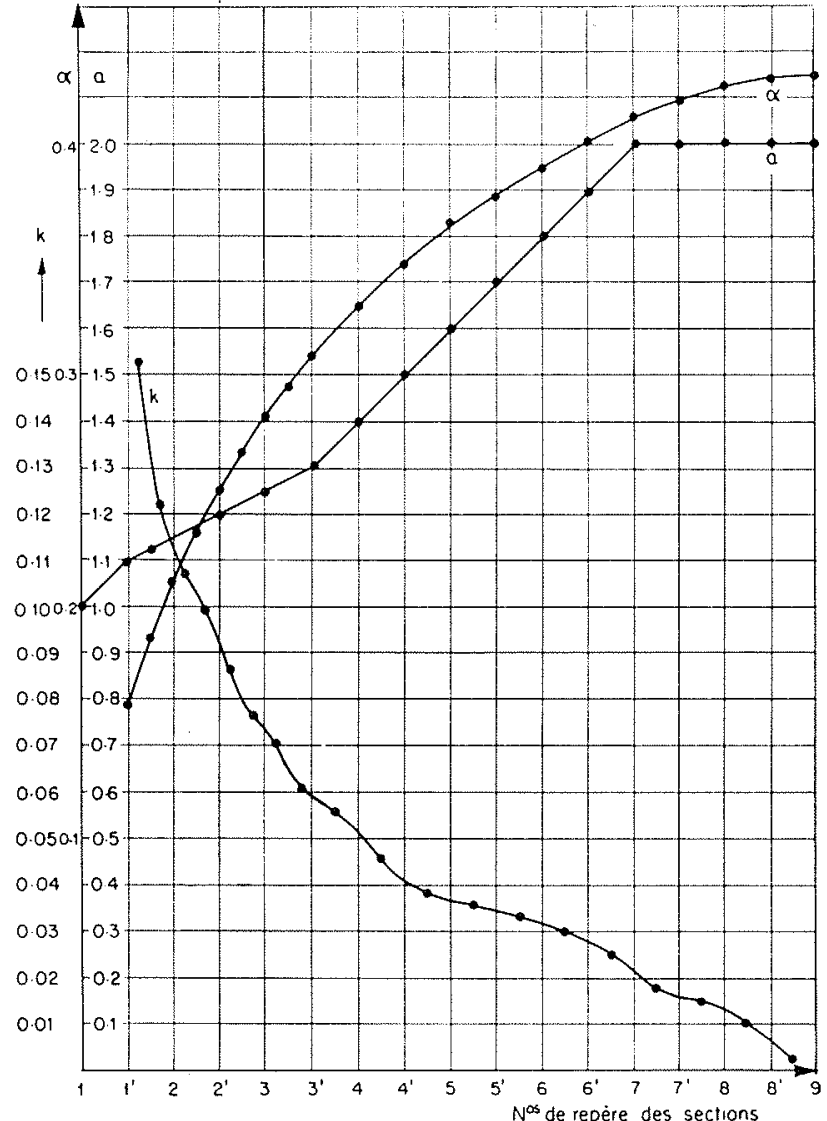

Fig. $20 a$

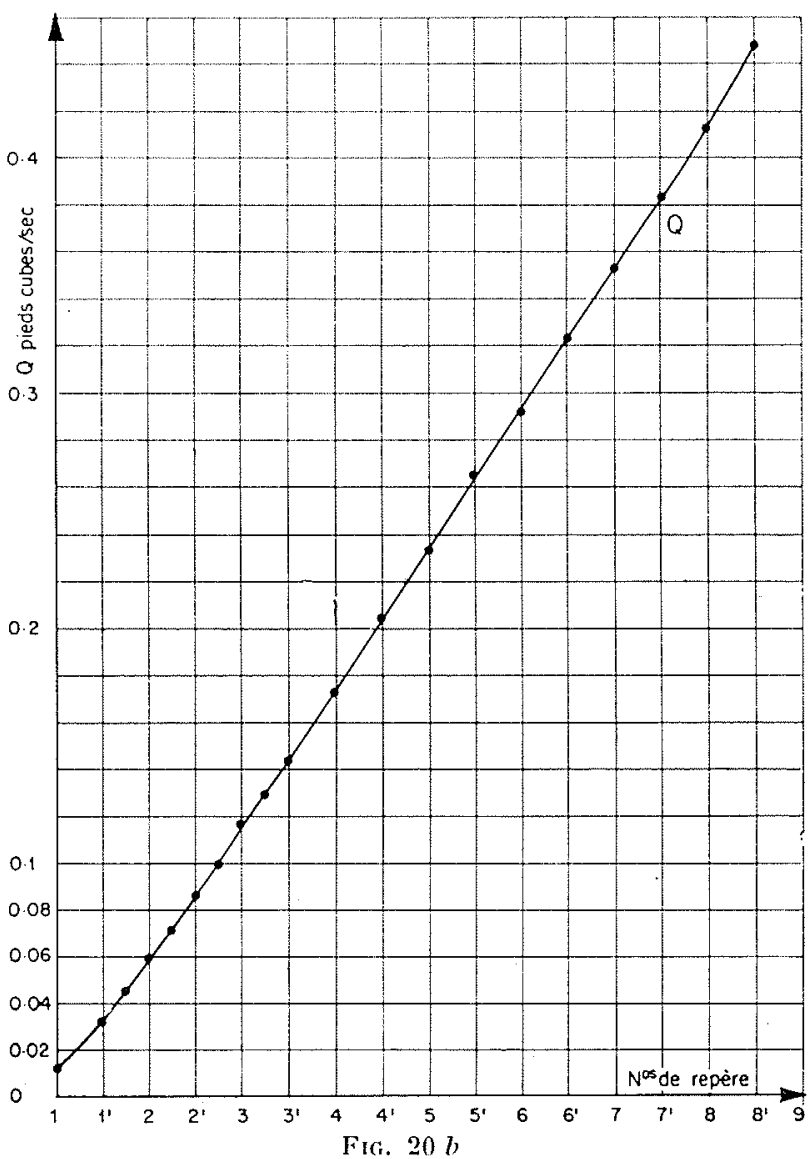




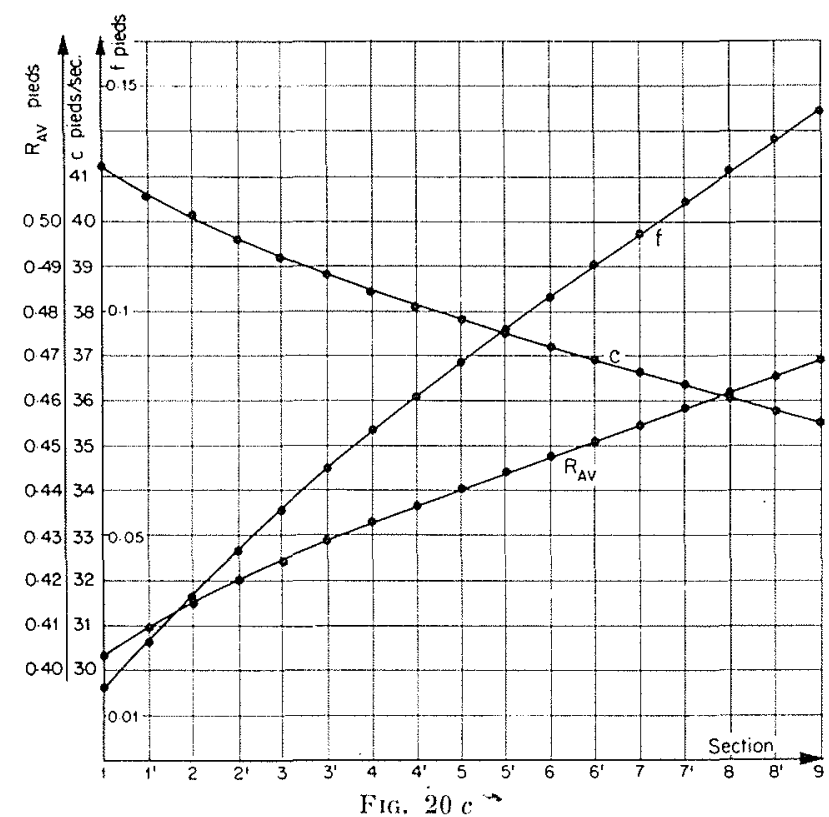

20-A. Nous avons donné la valeur calculée de la capacité $Q$ correspondant à l'écoulement de la volute pour chaque section sur la figure 20-B.

La valeur de $" c$ » est le facteur le plus inportant dans l'équation et se trouve définie lorsque la valeur de $V_{t}$ et une courbure de la volute permettant d'obtenir le rayon moyen $\mathrm{R}_{a v}$ ont été choisies.

Les valeurs de « $\mathrm{C} »$ calculées pour chaque section transversale, avec les valeurs de « $f$ » (fig. 11) et $\mathrm{R}_{a}$ sont indiquées sur la figure 20-C.

On commence le calcul de la volute à partir de la section la plus proche de la languette. Pour définir la constante « $C$ » dans l'équation (25), nous avons pris 0,000972 pied carré comme valeur de l'aire de section de la volute à la position $1^{\prime}$ et nous avons appliqué l'équation (25) entre la position $1^{\prime}$ et $\left(1^{\prime}\right)^{\prime}$.

Dans ce cas, nous avons les valeurs suivantes : $k=0,1532 \quad a=1,1 \quad \alpha=0,157 \quad c=40,475 \mathrm{pied} / \mathrm{s}$.

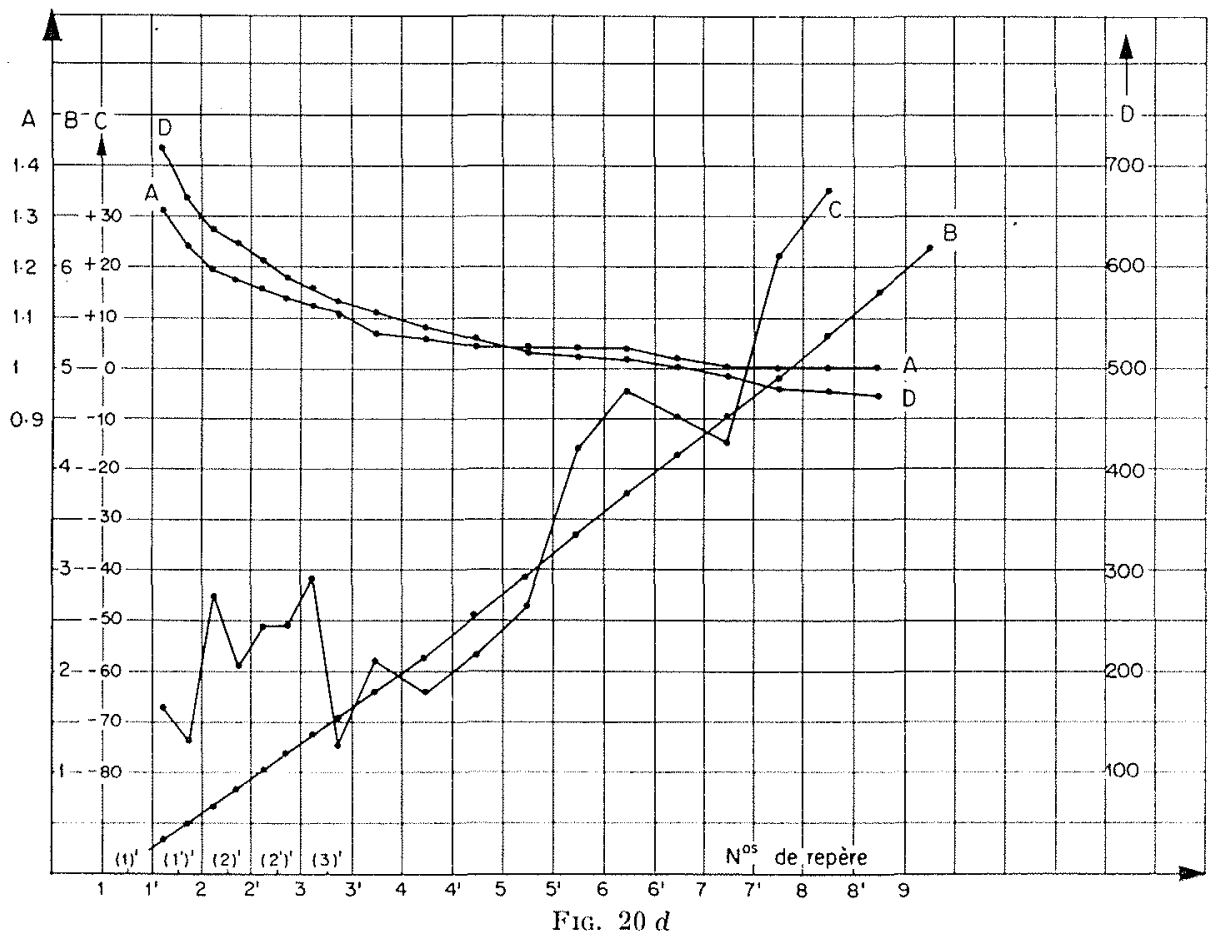

et divisons la volute en 21 sections avec des intervalles plus rapprochés près de la languette et plus espacés vers le còté extérieur de la volute; repérons ces sections en les numérotant de la manière suivante : $1-(1)^{\prime}-1^{\prime}-\left(1^{\prime}\right)^{\prime}-$ $2 \ldots$

Les positions des sections transversales choisies pour le calcul sont indiquées sur la figure 19.

Appliquons l'équation (24) à chaque section transversale séparément en utilisant les valeurs appropriées de $\alpha, k, a, c$, etc.

Les valeurs calculées de $\alpha$ et $k$ el les valeurs choisies pour « $a »$ sont indiquées sur la figure
Les valeurs calculées de $A, B$ et $D$ sont :

$$
\begin{aligned}
& A=\frac{2}{1-(k / a)}-1=\frac{2}{1-(0,1532 / 1,1)}-1=1,315 \\
& \mathrm{~B}=\frac{\mathrm{Q}_{1}-\left(\mathrm{Q} / \mathrm{Q}^{\prime} \pi\right) \cdot \alpha_{1} / a}{\mathrm{Q}_{n} / 2 \pi[1-(k / a)]} \\
& =\frac{0,0324-(0,475 / 2 \pi)(0,157 / 1.1)}{0.475 / 2 \pi[1-(0,1532 / 1.1)]}=0,331 \\
& \mathrm{D}=\frac{c}{\mathrm{Q}_{y} / 2 \pi\lceil 1-(k / \alpha)\rceil^{2}} \\
& =\frac{40,475}{0.475 / 2 \pi[1-(0,1532 / 1.1)]^{2}}=716
\end{aligned}
$$




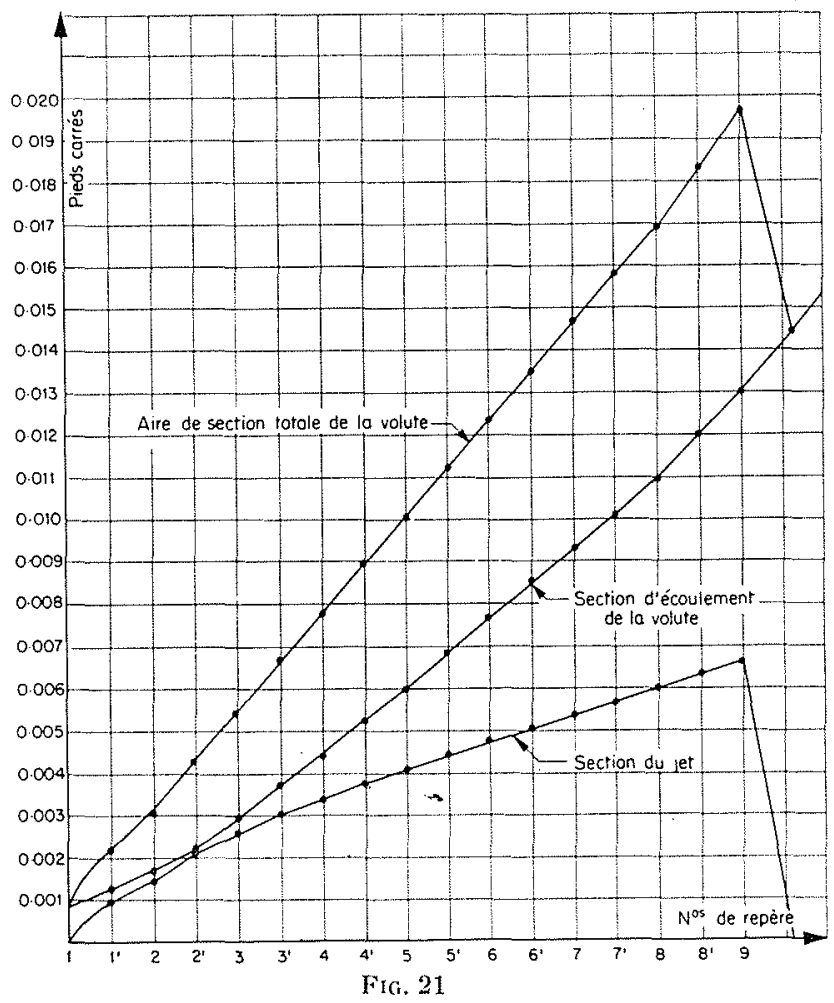

Pour trouver la valeur de 《C », qui est la constante dans l'équation (25), nous prenons la valeur de $\Omega=0,000972$ pied carré pour la position $1^{\prime}$ qui correspond à la valeur $\theta=0$. Dans ce cas, nous obtenons la valeur de la constante :

$$
\mathrm{C}=\frac{0.331^{2}}{0,000972}-\frac{716 \times 0,331}{1,315}=-67,2
$$

Comme chaque terme de l'équation (2) est connu, nous pouvons maintenant définir l'aire pour la position $\left(1^{\prime}\right)^{\prime}$ qui correspond à la valeur de $\theta=\pi / 16$ :

$$
\begin{aligned}
\Omega=\frac{1,315[(\pi / 16)+0,331]^{2}}{716[(\pi / 16)+0,331]-1,315 \times 67,2} \\
=0,001263 \text { pied carré }
\end{aligned}
$$

Etant donné que nous connaissons l'aire de la section transversale pour la position $\left(\mathbf{1}^{\prime}\right)^{\prime}$, nous pouvons appliquer à nouveau et de la même manière l'équation (25) entre les positions $\left(1^{\prime}\right)^{\prime}$ et 2 avec les nouvelles valeurs de $k ; a ; \alpha ; c ; \mathrm{Q}_{1}$ et $\mathrm{A}, \mathrm{B}, \mathrm{C}$ et $\mathrm{D}$ correspondant à la gamme de $\left(1^{\prime}\right)^{\prime}$ à 2. Les valeurs de $A, B, C$ et $D$ calculées de cette manière sont indiquées sur la figure 20-D et les valeurs numériques sont données dans le tableau IV.

Les résultats obtenus pour l'aire de section de la volute, du jet et de l'aire totale de la volute sont indiqués sur la figure 21 .

\section{Résultats de l'expérience}

Nous avons déterminé la répartition de la pression statique autour de la périphérie de la volute pour deux volumes différents :

$\mathrm{Q}=0.470$ pied cube $/ \mathrm{s}$ et $\mathrm{Q}=0,530 \mathrm{pied}$ cube $/ \mathrm{s}$,

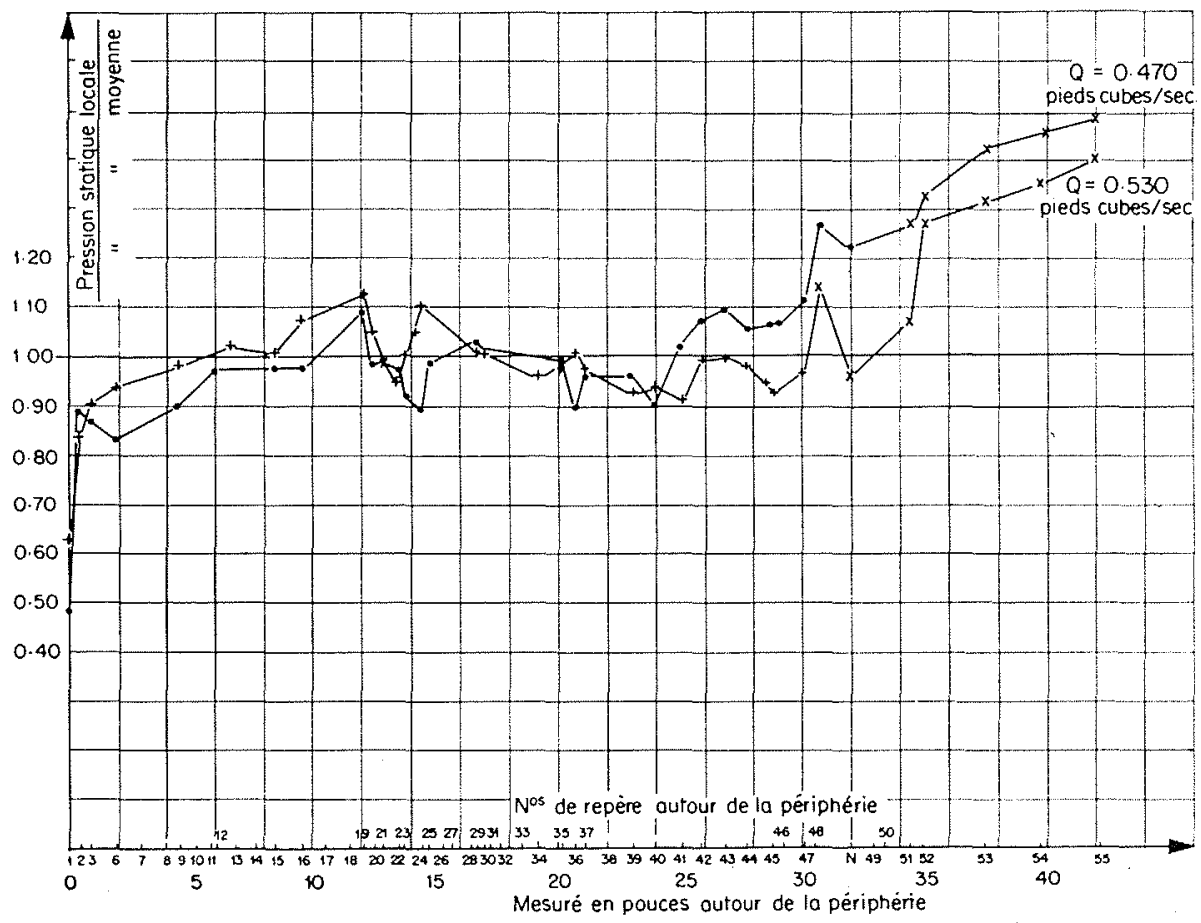

Fig. 22 


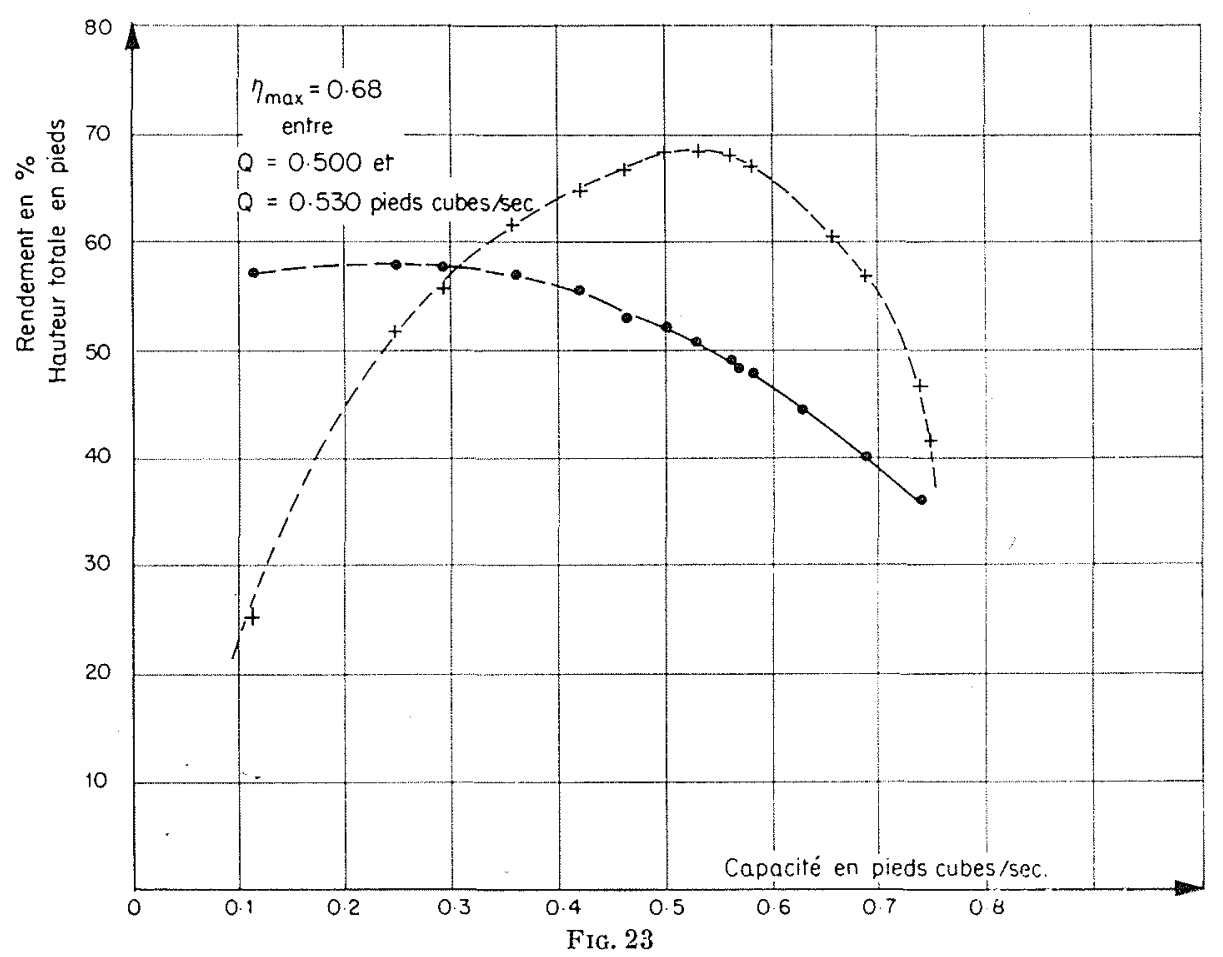

en mesurant les pressions statiques en 48 points différents comme indiqué sur la figure 15.

La répartition de la pression statique autour de la périphérie de la volute est indiquée sur la figure 22, sans dimensions, comme étant le rapport de la pression statique locale mesurée et de la moyenne des pressions statiques locales autour de la volute.

Le rendement de la pompe est supérieur à $68 \%$ pour une gamme allant de $Q=0,500$ à $\mathrm{Q}=0,530 \mathrm{pied}$ cube $/ \mathrm{s}$, et entre $\mathrm{Q}=0,410$ et $\mathrm{Q}=0,620$ pied cube $/ \mathrm{s}$, il est de plus de $64 \%$, autrement dit pour une gamme de 0,21 pied cube/s ou $40 \%$ de la capacité normale (fig. 23).

Entre le volume $Q=0.450 \mathrm{pied}$ cube $/ \mathrm{s}$ et $\mathrm{Q}=0,600 \mathrm{pied}$ cube/s, le rendement est supérieur à $66 \%$ pour une gamme de 0,150 pied cube $/ \mathrm{s}$, soit $29 \%$ de la capacitè normale.

Si l'on compare le rendement maximum obtenu avec cette volute de conception nouvelle, avec le rendement de la même pompe (mais avec une volute classique), on voit qu'il lui est supérieur de $2 \%$ et que la gamme des capacités correspondant à un rendement supérieur à $64 \%$ est de $40 \%$, tandis qu'elle n'est que de $27 \%$ avec la volute de conception classique.

\section{CONCLUSIONS}

1. La comparaison des figures 22 et 8 montre que la nouvelle conception donne une répartition des pressions statiques plus uniformes autour de la volute.

2. La comparaison des figures 23 et 10 montre que le rendement avec la nouvelle volute est de $2 \%$ plus élevé qu'avec la volute de conception classique.

3. Sur la figure 22, la fluctuation de la pression statique superposée à la courbe générale de la répartition des pressions statiques existe toujours, et il faudra faire de nouveaux essais pour définir sa cause et sa nature. Elle pourrait être due à l'oscillation du sens de la vitesse absolue pendant une révolution, si elle n'est pas due à de faibles erreurs d'exécution survenues au cours de la construction de la volute.

4. Pour le calcul de cette volute, nous avons choisi empiriquement certaines valeurs, particulicrement la composante tangentielle de la vitesse absolue à la sortie de la roue $\left(V_{l}\right)$ et il semble qu'un choix plus judicieux des premières données pourrait avoir pour résultat un rendement encore accru de la pompe. 
Tableau IV

\begin{tabular}{|c|c|c|c|c|c|c|c|c|c|c|c|c|}
\hline & $\begin{array}{c}Q_{1} \\
\text { en } \\
\text { pieds-cubes/sec }\end{array}$ & $\begin{array}{c}1 \\
\text { en } \\
\text { pouces }\end{array}$ & $\begin{array}{c}\mathrm{R}_{\mathrm{alr}} \\
\text { en } \\
\text { pieds }\end{array}$ & $a$ & $\alpha$ & $k$ & $\mid \begin{array}{c}c \\
\text { en } \\
\text { pieds/sec }\end{array}$ & $A$ & $B$ & C & D) & $\begin{array}{c}\Omega \\
\text { en } \\
\text { pieds carlés }\end{array}$ \\
\hline 1 & & 0,200 & 0,404 & & & & 41,20 & & & & & \\
\hline$(1)^{\prime}$ & & & & 1 & & & & & & & & \\
\hline $1^{\prime}$ & 0,0324 & 0,320 & 0,410 & 1,05 & & & \multirow{2}{*}{40,60} & & & & & 0,000972 \\
\hline$\left(1^{\prime}\right)^{\prime}$ & 0,0451 & & & 1,1 & 0,157 & 0,1532 & & 1,315 & 0,331 & $-67,2$ & 716 & 0,001263 \\
\hline 2 & 0,0593 & 0,440 & 0,415 & 1,125 & 0,187 & 0,1226 & \multirow{2}{*}{40,10} & 1,24 & 0,488 & $-73,8$ & 667 & 0,001422 \\
\hline$(2)^{\prime}$ & 0,0713 & & & 1,15 & 0,211 & 0,1070 & & 1,19 & 0,663 & $-45,2$ & 637 & 0,001775 \\
\hline $2^{\prime}$ & 0,0865 & 0,560 & 0,420 & 1,175 & 0,232 & 0,0996 & \multirow{2}{*}{39,60} & 1,175 & 0,814 & -59 & 624 & 0,00214 \\
\hline$\left(2^{\prime}\right)^{\prime}$ & 0,1006 & & & 1,200 & 0,250 & 0,0868 & & 1,155 & 1,011 & -51 & 606 & 0,00251 \\
\hline 3 & 0,1174 & 0,670 & 0,424 & 1,225 & 0,267 & 0,0764 & \multirow{2}{*}{39,20} & 1,135 & 1,188 & -52 & 589 & 0,00288 \\
\hline$(3)^{\prime}$ & 0,1294 & & & 1,250 & 0,282 & 0,0710 & & 1,120 & 1,400 & -42 & 578 & 0,00327 \\
\hline $3^{\prime}$ & 0,1435 & 0,780 & 0,4285 & 1,275 & 0,295 & 0,0612 & 38,85 & 1,10 & 1,540 & -75 & 565 & 0,00370 \\
\hline 4 & 0,1732 & 0,880 & 0,433 & 1,300 & 0,308 & 0,0559 & 38,45 & 1,07 & 1,800 & -58 & 554 & 0,00444 \\
\hline $4^{\prime}$ & 0,2045 & 0,975 & 0,437 & 1,400 & 0,330 & 0,0458 & 38,10 & 1,058 & 2,13 & -64 & 540 & 0,005225 \\
\hline 5 & 0,2332 & 1,065 & 0,440 & 1,500 & 0,348 & 0,0382 & 37,80 & 1,041 & 2,54 & -57 & 530 & 0,006015 \\
\hline $5^{\prime}$ & 0,2652 & 1,150 & 0,444 & 1,60 & 0,363 & 0,0356 & 37,50 & 1,038 & 2,92 & -47 & 518,5 & 0,006820 \\
\hline 6 & 0,2932 & 1,235 & 0,4475 & 1,70 & 0,377 & 0,0331 & 37,20 & 1,037 & 3,34 & -16 & 513,5 & 0,00765 \\
\hline $6^{\prime}$ & 0,3234 & 1,320 & 0,4510 & 1,80 & 0,390 & 0,0305 & 36,90 & $1,(1035$ & 3,73 & -5 & 507 & 0,00844 \\
\hline 7 & 0,3535 & 1,405 & 0,4545 & 1,90 & 0,402 & 0,0254 & 36,65 & 1,018 & 4,12 & -10 & 501 & 0,00930 \\
\hline $7^{\prime}$ & 0,3833 & 1,490 & 0,4580 & 2 & 0,412 & 0,0178 & 36,35 & 1,00 & 4,52 & -15 & 492 & 0,01010 \\
\hline 8 & 0,4132 & 1,575 & 0,4616 & 2 & 0,419 & 0,0153 & 36,05 & 1,00 & 4.89 & +22 & 478 & 0,01094 \\
\hline $8^{\prime}$ & 0,4482 & 1,660 & 0,4651 & 2 & 0,425 & 0,0104 & 35,75 & 1,00 & 5,28 & +35 & 476 & 0,01199 \\
\hline 9 & & 1,745 & 0,4685 & 2 & 0,429 & 0,0012 & 35,50 & 1,00 & 5,73 & & 472 & 0,01305 \\
\hline
\end{tabular}

\section{REFERENCES}

(1) R. C. Broer and R. T. KNapl. - Ixperimental determination of the flow characteristics in the volute of centrifugal pumps. A.S.M.E., November 1936.

(2) R. D. Bowermas. - Effect of the volute on performance of a centrifugal pump. Calif. Institute of Technology, Hydrodynamics Laboratory. Report $n^{\circ}$ E-19.7, mars 1955.

(3) C. S. Dearin. - Study of the ontflow to the volute from a centrifugal pump impeller with special reference to velocity distribution. Unpublished Report to D.S.I.R. 